\title{
Neuronal post-developmentally acting SAX-7S/L1CAM can function as cleaved fragments to maintain neuronal architecture in $C$. elegans
}

\author{
V.E. Desse ${ }^{1}$, C.R. Blanchette ${ }^{2}$, P. Perrat ${ }^{2}$, C.Y. Bénard ${ }^{1,2, *}$
}

\author{
Affiliations: \\ 1 Université du Québec à Montréal \\ Department of Biological Sciences \\ 141 President Kennedy Avenue \\ Montréal, QC H2X 1Y4, Canada \\ E-mail addresses: \\ VED: desse.virginie@uqam.ca \\ CRB: cblanchette@brandeis.edu \\ PP: paola.perrat@egenesisbio.com \\ ${ }^{2}$ University of Massachusetts Medical School \\ Department of Neurobiology \\ 364 Plantation St, Worcester \\ MA 01605, USA \\ CYB: benard.claire@uqam.ca \\ ${ }^{*}$ Corresponding author: \\ Claire Bénard \\ Université du Québec à Montréal \\ Department of Biological Sciences \\ 141 President Kennedy Avenue \\ Montréal, QC H2X 1Y4, Canada \\ E-mail: benard.claire@uqam.ca; claire.benard@umassmed.edu \\ Phone: 1-514-987-3000, extension -6192
}




\section{ABSTRACT}

Whereas remarkable advances have uncovered mechanisms that drive nervous system assembly, the processes responsible for the lifelong maintenance of nervous system architecture remain poorly understood. Subsequent to its establishment during embryogenesis, neuronal architecture is maintained throughout life in the face of the animal's growth, maturation processes, the addition of new neurons, body movements, and aging. The C. elegans protein SAX-7, homologous to the vertebrate L1 protein family, is required for maintaining the organization of neuronal ganglia and fascicles after their successful initial embryonic development. To dissect the function of sax-7 in neuronal maintenance, we generated a null allele and sax-7S-isoform-specific alleles. We find that the null sax-7(qv30) is, in some contexts, more severe than previously described mutant alleles, and that the loss of sax-7S largely phenocopies the null, consistent with sax-7S being the key isoform in neuronal maintenance. Using a sfGFP::SAX-7S knock-in, we observe sax-7S to be predominantly expressed across the nervous system, from embryogenesis to adulthood. Yet, its role in maintaining neuronal organization is ensured by post-developmentally acting SAX$7 S$, as larval transgenic sax-7S(+) expression alone is sufficient to profoundly rescue the null mutants' neuronal maintenance defects. Moreover, the majority of the protein SAX-7 appears to be cleaved, and we show that these cleaved SAX-7S fragments together, not individually, can fully support neuronal maintenance. These findings contribute to our understanding of the role of the conserved protein SAX-7/L1CAM in long-term neuronal maintenance, and may help decipher processes that go awry in some neurodegenerative conditions.

Running title: SAX-7S in neuronal maintenance

62

63

Keywords: neuronal maintenance, lifelong, L1, sax-7, Ig, cleavage 


\section{INTRODUCTION}

An important yet poorly understood question of neurobiology is how the organization of neural circuits is maintained over a lifetime to ensure their proper function. Largely established during embryogenesis, the architecture of the nervous system needs to persist throughout life in the face of the animal's growth, the addition of new neurons, maturation processes, body movements, and aging. Whereas significant progress has been made in understanding the processes driving neuronal development, little is known about the mechanisms ensuring lifelong maintenance of nervous system architecture and function.

Research using $C$. elegans has uncovered a number of immunoglobulin (Ig) superfamily molecules required for the long-term maintenance of neuronal architecture (Benard and Hobert, 2009). These include the large extracellular protein DIG-1 (Benard et al., 2006; Johnson and Kramer, 2012), the small two-lg domain proteins ZIG-3, ZIG-4, and ZIG-10 (Aurelio et al., 2002; Benard and Hobert, 2009; Benard et al., 2012; Cherra and Jin, 2016), the ectodomain of the FGF receptor EGL-15 (Bülow et al., 2004), as well as SAX-7 (Pocock et al., 2008; Sasakura et al., 2005; Wang et al., 2005; Zallen et al., 1999; Zhou et al., 2008). Here, we further the investigation of SAX-7/L1CAM's role in the lifelong maintenance of neuronal architecture.

SAX-7 is an evolutionary conserved transmembrane cell adhesion molecule homologous to mammalian L1CAM (Chen et al., 2001; Hortsch, 2000; Hortsch et al., 2014). In C. elegans, SAX-7 exists as two main isoforms, a long isoform SAX-7L and a short isoform SAX-7S. These two isoforms are identical for their intracellular tail, transmembrane domain (TM), and most of their extracellular region including five identical fibronectin type III domains (FnIII), and four Ig-like domains. They differ in the N-terminal extracellular region, where SAX7S has four Ig domains ( $\lg 3-6)$, whereas SAX-7L has six Ig domains $(\lg 1-6)$. Transgenes of SAX-7S, but not of SAX-7L, rescue the defects of sax-7 loss-of-function mutants, indicating that the SAX-7S isoform is central to sax-7 functions (Pocock et al., 2008; Ramirez-Suarez et al., 2019; Sasakura et al., 2005; Wang et al., 2005). Vertebrate proteins of the SAX-7/L1CAM family include L1CAM, NrCAM, CHL1, and Neurofascin (Brummendorf et al., 1998; Brummendorf and Rathjen, 1996; Haspel and Grumet, 2003; Hortsch, 2000; Hortsch et al., 2014).

sax-7/L1CAM is well known to contribute to the development of distinct neurons in $C$. elegans. It is involved in dendrite development and axon guidance (Cebul et al., 2020; Chen et al., 2019; Diaz-Balzac et al., 2015; Diaz-Balzac et al., 2016; Dong et al., 2013; Heiman and Pallanck, 2011; Ramirez-Suarez et al., 2019; Salzberg et al., 2013; Schafer and Frotscher, 2012; Sherry et al., 2020; Yip and Heiman, 2018; Zhao et al., 1998; Zhu et al., 2017). In flies and mammals, homologues of sax-7 function in neuronal migration, axon guidance, and synaptogenesis (Bieber et al., 1989; Godenschwege et al., 2006; Hall and Bieber, 1997; Rougon and Hobert, 2003; Sonderegger et al., 1998). In humans, mutations in L1CAM severely impair neuronal development, leading to disorders collectively referred to L1 or CRASH syndrome for corpus callosum hypoplasia, mental retardation, aphasia, spastic paraplegia and hydrocephalus (Fransen et al., 1997; Hortsch et al., 2014).

Besides their roles in neuronal development, SAX-7/L1CAM family members also function in the mature nervous system to preserve neuronal organization. In C. elegans, sax- 
1097 is required for maintaining neuronal organization well after development is completed, as

110 specific neuronal structures that initially develop normally in sax-7 mutant animals, later

111 become disorganized. For instance, in sax-7 mutants, a subset of axons within the ventral

112 nerve cord, which developed normally during embryogenesis, become displaced to the

113 contralateral fascicle during the first larval stage; and neurons within embryonically

114 established ganglia become progressively disorganized by late larval stages and adulthood

115 in sax-7 mutants (Pocock et al., 2008; Sasakura et al., 2005; Wang et al., 2005; Zallen et al.,

116 1999; Zhou et al., 2008). Such post-developmental neuronal disorganization displayed by

117 sax-7 mutant animals can be prevented if animals are paralyzed (Pocock et al., 2008;

118 Sasakura et al., 2005), indicating that the mechanical stress from body movements

119 contributes to perturbing neuronal architecture in these mutants. In mammals, roles for L1

120 family members in the adult nervous system have been revealed as well through the study of

121 conditional knockouts. Adult-specific knockout of neurofascin affects rats behavior and alters

122 the axon initial segment in mice (Kriebel et al., 2011; Zonta et al., 2011); knockout of L1CAM

123 specifically in the adult mouse brain leads to behavioral deficits and synaptic transmission

124 changes (Law et al., 2003); and CHL1 conditional depletion in a subtype of forebrain neurons

125 in mice leads to defects in working memory duration (Kolata et al., 2008). Thus, L1CAM family

126 proteins contribute to preserving the functionality of the mammalian adult nervous system.

127 Despite the evolutionarily conserved importance of SAX-7/L1CAM, its role in the long-

128 term maintenance of the neuronal architecture remains unclear. In order to better understand

129 how SAX-7/L1CAM participates in neuronal maintenance, here we have generated and

130 characterized a null allele of sax-7, tested the temporal requirements for sax-7S neuronal

131 maintenance function, determined the endogenous expression pattern of SAX-7S, and

132 assessed the function of SAX-7S cleavage products in neuronal maintenance. Our results

133 further our understanding of the roles of the evolutionarily conserved molecule SAX-7/L1CAM

134 in the lifelong persistence of neuronal organization and function.

\section{RESULTS}

140

141

142

\section{Molecular analysis of previous sax-7 mutant alleles}

143

The interpretation of previous structure-function analyses for sax-7 was limited by the lack of a clear null mutation for the gene. In particular, the existence of gene product in sax-7(nj48), an allele reported to be a complete loss-of-function of the gene sax-7, has not been fully assessed. We examined sax-7 transcripts by RT-PCR for nj48, as well as for other sax-7 mutant alleles, including the sax-7L-specific alleles eq2 and $n j 53$, and two alleles that affect both sax-7 isoforms, tm1448 and eq1 (Fig. 1A,B). We detected transcripts corresponding to all isoforms of sax-7 in all mutants tested (Fig. 1C; all RT-PCR products were verified by sequencing), except when the primer targets a sequence that is deleted by a given mutation. In particular, transcript was detected in nj48 mutants, using four different primer pairs (Fig. 1C), indicating that $n j 48$ is not a null allele. 
We also carried out western blots to characterize the expression of the protein SAX-7 in sax-7(nj48) and other mutant alleles (Fig. 1D). To detect SAX-7, we used a purified antibody generated against the cytoplasmic tail of SAX-7 (Chen et al., 2001). In wild-type extracts, we detect five protein bands of $\sim 190 \mathrm{kDa}, 150 \mathrm{kDa}, 60 \mathrm{kDa}, 40 \mathrm{kDa}$ and $28 \mathrm{kDa}$ that are absent in the control eq1, in which the epitope-containing region of SAX-7 is deleted, and in a newly generated deletion allele qv30, in which the entire locus of sax-7 is absent (see below). The $190 \mathrm{kDa}$ band (Fig. 1D, blue arrow) and the $150 \mathrm{kDa}$ band (Fig. 1D, green arrow) correspond to the predicted SAX-7L and SAX-7S full-length protein, respectively, as previously reported (Chen et al., 2001; Sasakura et al., 2005; Wang et al., 2005). Two bands at $60 \mathrm{kDa}$ and $28 \mathrm{kDa}$ appear to be cleavage products. The $60 \mathrm{kDa}$ band (Fig. 1D, red arrow) is likely the C-terminal fragment resulting from proteolytic cleavage of SAX-7 at the serine protease site in the $3^{\text {rd }} \mathrm{FnIII}$ domain (Fig. 1B). This cleavage site is conserved in vertebrate L1 proteins (Faissner et al., 1985; Haspel and Grumet, 2003; Hortsch, 1996, 2000; Kalus et al., 2003; Lutz et al., 2017; Lutz et al., 2012; Matsumoto-Miyai et al., 2003; Mechtersheimer et al., 2001; Nayeem et al., 1999; Sadoul et al., 1988; Schafer and Altevogt, 2010; Silletti et al., 2000; Xu et al., 2003). The 28 kDa band (Fig. 1D, black arrow), which runs as a doublet, is likely the predicted C-terminal fragment resulting from the proteolytic cleavage of SAX-7 at the proximal-transmembrane extracellular site (TM site, Fig. 1B). Similar metalloprotease cleavage sites have been reported in vertebrate L1CAM proteins (Beer et al., 1999; Gutwein et al., 2003; Haspel and Grumet, 2003; Jafari et al., 2010; Kalus et al., 2003; Kiefel et al., 2012; Linneberg et al., 2019; Maretzky et al., 2005; Maten et al., 2019; Matsumoto-Miyai et al., 2003; Mechtersheimer et al., 2001; Naus et al., 2004; Nayeem et al., 1999; Riedle et al., 2009; Sadoul et al., 1988; Schafer and Altevogt, 2010; Tatti et al., 2015; Xu et al., 2003; Zhou et al., 2012). Finally, a $40 \mathrm{kDa}$ band is detected in the wild type, but is absent in the controls (Fig. 1D), suggesting yet another form of SAX-7 (also recently indicated in WormBase). Noteworthy, we find that the level of the full-length SAX-7L protein is higher than the fulllength SAX-7S, and that most SAX-7 is detected as a cleaved form. In particular, the serine protease-cleavage product $(\sim 80 \%)$ is most abundant (only the C-terminal fragment of the serine protease cleavage can be detected, as epitope located in the C-terminus). In contrast, the proximal-TM cleavage site product is less abundant (Fig. 1D). Importantly, in extracts of nj48 mutants, two forms of SAX-7 protein were detected, which were absent in controls: a 40 kDa band (Fig. 1D, indicated by a question mark), and a $140 \mathrm{kDa}$ band, likely a truncated form of SAX-7 protein (Fig. 1D, black arrowhead). Thus, sax-7 transcript and SAX-7 protein are detected in extracts of sax-7(nj48) mutants, revealing that $n j 48$ is not a null allele.

186

\section{Generation of a sax-7 null and sax-7S-specific mutant alleles}

To generate a null allele of sax-7, we used CRISPR-Cas9 technology and deleted the entire locus of the sax-7 gene. Two targets were used, one on the $1^{\text {st }}$ exon of sax- $7 L$ and one on the last exon of sax-7 (exon 17 and 14 of the long and short isoform, respectively), resulting a 19,972 bp deletion (Figs. 1A, S1A). This new mutant, named sax-7(qv30), is a clear null allele of sax-7 and was verified by multiple PCRs, sequencing, and western blot (Fig. 1D). sax-7(qv30) null mutants are viable and have a somewhat reduced brood size, but their egg laying and embryonic viability are normal (Fig. S2). 
We also generated sax-7S-isoform specific alleles, as this isoform has been found to be functionally important. Using CRISPR-Cas9 technology, we targeted the $1^{\text {st }}$ exon of sax$7 S$ specifically (in a region corresponding to an intron in sax-7L) and obtained two small sax$7 S$-specific insertion alleles, qv25 and $q v 26$, both predicted to be strong loss-of-function alleles of sax-7S. qv25 has a 47 bp insertion and qv26, a 36 bp insertion (Fig. S1B-C). Both alleles disrupt the sax-7S export signal peptide sequence, likely disturbing SAX-7S protein synthesis. As a further consequence of the qv25 insertion, a stop codon is generated in the open reading frame of sax-7S (Fig. S1B). At the protein level, using the antibody against the SAX-7 cytoplasmic tail (Chen et al., 2001), as expected we detected no full-length SAX-7S in extracts of these mutants, while full-length SAX-7L was detected (190 kDa band; Fig. 1D). As a note, it appears that when SAX-7S is affected, as in $q v 25$ and $q v 26$, the $60 \mathrm{kDa}-\mathrm{C}$ terminal-serine protease-cleavage product is less abundant than in wild type or sax-7Lspecific mutants eq2 and nj53 (60 kDa band; Fig. 1D). This was consistently observed in all of the western blots done using either mixed worm populations or 100 L4 worms ( $\geq 3$ independent repeats in each case). It thus appears that a large proportion of the C-terminal serine protease cleavage product may originate from cleavage of SAX-7S protein specifically.

\section{Phenotypic characterization of new sax-7 mutants}

We characterized the phenotypic consequences of the complete loss of sax-7 function in sax7(qv30) mutants in neuronal maintenance. As a measure of head ganglia organization, we examined two pairs of head chemosensory neurons (ASH and $\mathrm{ASI}$ ) from the $2^{\text {nd }}$ larval stage to adulthood, as previously done for other mutants (Benard et al., 2009; Benard et al., 2012; Benard et al., 2006). The soma of these neurons are located in the lateral head ganglia and their axons project into the nerve ring. We visualized these 4 neurons using the fluorescent Psra-6::gfp or Psra-6::DsRed2 and noted the relative position of the ASH/ASI cell bodies with respect to the nerve ring. We found that head ganglia organization is normal in $2^{\text {nd }}$ larval stage qv30 null mutants, but that it becomes progressively disorganized by the $4^{\text {th }}$ larval stage, worsening into adulthood (Fig. 2A). Similar disorganization of ASH/ASI has been described in nj48 mutant adults (Benard et al., 2012).

We also examined the precise axon position of the two pairs of bilateral interneurons (PVQ and PVP) in the ventral nerve cord, labelled by the reporters Psra-6::DsRed2 and Podr$2:: c f p$, respectively. These axons are normally positioned in freshly hatched $1^{\text {st }}$ stage larvae of qv30 mutants, indicating that they had extended normally along the ventral nerve cord during embryogenesis. However, compared to wild type where the PVQ and PVP axons remain well positioned in virtually all animals $(94 \%, n=117)$, in sax-7(qv30) mutants these axons later fail to maintain this positioning and inappropriately flip-over to the other side of the ventral nerve cord in $37.5 \%$ of $q v 30$ animals $(n=80)$, which is similar to $n j 48$ mutants (Benard et al., 2012; Pocock et al., 2008).

Other aspects of neuroanatomy of qv30 mutants were more severe than nj48 mutants. For instance, we observed retrovesicular ganglia organization by visualizing the neurons AIY and AVK (using reporters Pttx-3::mCherry and Pflp-1::gfp, respectively) and found that $85 \%$ of 1-day adult qv30 mutant animals display disjointed AIY and AVK soma, compared to $70 \%$ in nj48 mutants (Fig. 2B). Also, using Dil staining we found that the position of the soma of 
nj48 mutants, at the $4^{\text {th }}$ larval stage (Fig. 2C). Thus, while nj48 is a strong allele displaying similar penetrance to the null allele $q v 30$ in some neuronal contexts, its loss of function is partial and less severe than the null qv30 in other neuronal contexts.

\section{SAX-7S is required for neuronal maintenance}

sax-7S, but not sax-7L, has previously been found to be sufficient to rescue neuronal maintenance defects in sax-7(nj48) mutants (Pocock et al., 2008; Sasakura et al., 2005). We verified whether sax-7S is also sufficient to rescue such defects in the sax-7(qv30) null mutants, by generating transgenic qv30 null mutant animals carrying wild-type copies of sax$7 S(+)$ expressed neuronally [using the transgenes Punc-14::sax-7S(+) and Prab-3::sax$7 S(+)$ ]. We found that $q v 30$ transgenic animals were profoundly rescued for head ganglia disorganization (Fig. 2A). On the other hand, wild-type sax-7L(+) did not rescue qv30 transgenic mutant animals (transgene Punc-14::sax-7L(+); Fig. 2A), similar to findings using the allele nj48 (Pocock et al., 2008; Sasakura et al., 2005). This is consistent with the absence of defects in sax-7L-specific mutants eq2 and nj53 (Benard et al., 2012; Sasakura et al., 2005). Altogether, these results further demonstrate that sax-7S mediates neuronal maintenance function.

To directly assess the phenotypic consequences of specifically disrupting sax-7S, we analyzed neuronal maintenance defects of the newly generated sax-7S-specific mutants qv25 and qv26 (Figs. 1A, 2A). We found that the severity of their defects is similar to qv30 null mutant animals. For instance, the head ganglia of $q v 25$ and $q v 26$ animals become disorganized from the $4^{\text {th }}$ larval stage onwards, similar in penetrance and expressivity to the qv30 null mutants (Fig. 2A). Also, the soma of retrovesicular ganglion neurons AIY and AVK become disorganized from the $4^{\text {th }}$ larval stage in qv25 mutants, similar to qv30 mutants (Fig. 2B). Finally, the soma of tail neurons PHA and PHB get displaced from the $4^{\text {th }}$ larval stage onwards in qv25 mutants, similar to qv30 mutants (Fig. 2C). Thus, the specific disruption of sax-7S leads to neuronal maintenance defects that are similar to those resulting from the complete loss of sax-7 (deleting both sax-7S and sax-7L), confirming the key role of SAX-7S in the maintenance of neuronal architecture.

\section{Post-developmental expression of sax-7S is sufficient for maintaining neuronal} architecture

271

Although the ventral nerve cord and head ganglia assemble during embryogenesis, sax7(qv30) null mutants manifest ventral nerve cord flip-over defects during larval development, and head ganglia become disorganized by late larval stages, progressively worsening into adulthood. The appearance of defects in sax-7 mutants could in theory result from either (a) undetected embryonic neuronal development defects that later worsen as the animal grows and moves, or (b) deficient neuronal maintenance during larval and adult stages. To distinguish between these possibilities, we carried out rescue assays of qv30 null mutants with wild-type sax-7S(+) copies expressed under the control of an inducible heat shock promoter, which drives expression in neurons and other tissues (Fire et al., 1990; Jones et al., 1986; Stringham et al., 1992). For this, we generated transgenic qv30 animals carrying the transgene Phsp16.2::sax-7S(+) as an extrachromosomal array. All animals were kept at 
shock treatments (Fig. 3A). The organization of the ASI and ASH head ganglia neurons was examined in 1-, 2-, 3-, 4-, and 5-day old adults. We controlled for head ganglia organization in the strains grown continuously at $15^{\circ} \mathrm{C}$ being indeed (a) normal in wild-type animals; (b) defective in qv30 mutants; (c) not rescued in the absence of heat shock, in transgenic qv30 animals carrying the transgene [Phsp-16.2::sax-7S(+)], indicating that the transgene is not expressed without heat shock; and (d) defective in qv30 non-transgenic control siblings under the same conditions (Fig. 3B).

To determine the temporal requirement in sax-7 function, we heat shocked $1^{\text {st }}(\mathrm{L} 1)$ or $3^{\text {rd }}(\mathrm{L} 3)$ larval stage animals that had otherwise been grown at $15^{\circ} \mathrm{C}$, and examined head ganglia organization at days 1, 2, 3, 4, and 5 of adulthood (Fig. 3A). Wild-type animals, qv30 mutants, transgenic qv30 animals carrying the transgene [Phsp-16.2::sax-7S(+)], and their qv30 non-transgenic siblings, were analyzed in parallel. An additional control consisted of heat-shock treatment alone, in the absence of the transgene, which did not modify the defects of sax-7 mutants (head ganglia are similarly disorganized in $q v 30$ animals whether heat shocked or not; Fig. 3B). Also, heat shock did not alter head ganglia organization in wild-type animals (Fig. 3B). In contrast, when transgenic qv30 animals carrying the transgene Phsp16.2::sax-7S(+) were heat shocked at L1, or even as late as $L 3$, their neuronal organization was profoundly rescued. This rescue by heat shock-induced expression of sax$7 S(+)$ is dependent on the presence of the transgene, as non-transgenic control siblings (which grew side by side with qv30 transgenics) were not rescued (Fig. 3B). Together, these results indicate that wild-type activity of sax-7S provided as late as the $3^{\text {rd }}$ larval stage is sufficient for it to function in the maintenance of neuronal architecture. Thus, sax-7S can function post-developmentally to maintain the organization of embryonically developed neuronal architecture. Moreover, we found that the rescue of $q v 30$ mutants following induction of sax-7S(+) is more profound in younger adults (days 1 to 3 ), as compared to older adults (days 4 and 5, Fig. 3B). By day 5 of adulthood, more than 6 days have passed after heat shock-induced expression of Phsp16.2::sax-7S(+), suggesting that de novo expression of sax-7S may be required to ensure its maintenance function during adulthood.

\section{Endogenous sax-7S is expressed in neurons}

The sax-7 gene is expressed strongly and broadly across the nervous system, as visualized with a fosmid (Sarov et al., 2012) where both the short and long isoforms are tagged with gfp (Fig. 4A; (Ramirez-Suarez et al., 2019)). To elucidate the expression pattern of sax-7S, we used CRISPR-Cas9 technology to tag the sax-7S isoform specifically with sfgfp at its endogenous genomic locus (Fig. 4B). We targeted the end of the $1^{\text {st }}$ exon of sax-7S in a precise region that corresponds to intron 4 of sax-7L, and inserted sfgfp, preceded by sax7S-signal-peptide coding sequence (sfGFP::SAX-7S; Figs. S1D, 4B). This knock-in allele of sax-7, named qv31, which was verified by sequencing (Fig. S1D), does not affect overall morphology or behavior, and head ganglia organization of qv31 animals is normal $(n=84,2 \%$ defects, examined at 1 -day adult by Dil staining, similar to wild type with $0 \%$ defects, $n=76$ ).

To characterize the temporal and spatial expression pattern of qv31 sfGFP::SAX-7S, we used conventional as well as confocal fluorescence microscopy with spectral unmixing. sfGFP::SAX-7S is seen most predominantly and abundantly across the nervous system, where it is observed in virtually all neuron of head and tail ganglia, the ventral nerve cord, as 
327 well as in isolated neurons located along the body wall (e.g. HSN near the vulva, and the 328 PVM post-deirid neuron). Expression of sfGFP::SAX-7S in neurons is first observed in 329 embryos (Fig. S3A), and persists throughout larval stages (Fig. 4B) and adulthood, including 330 in 5- and 8- day adults (Fig. 4B). While virtually all neurons express SAX-7S, differences in 331 the level of expression are observed among neurons. sfGFP::SAX-7S is also occasionally 332 detected in other cell types, such as in epidermal cells of the developing vulva and the uterus at the L4 stage, but not in adults (Fig. S3B). In sum, SAX-7S appears to be transiently and weakly expressed in developing cells of the epidermis, but its expression is strongest and sustained in virtually all neurons from embryogenesis to adulthood.

As a note, previous reports where sax-7 (both $L$ and $S$ indistinctly) was tagged intracellularly reported SAX-7 protein signal in axons, dendrites or the plasma membrane (Chen et al., 2001; Ramirez-Suarez et al., 2019; Wang et al., 2005). Here, the sfGFP::SAX$7 S$ signal in qv31 animals appears to be peri-nuclear in neuronal cell bodies, which is surprising for a transmembrane protein, and is likely artifactual. Indeed, in our effort to exclusively tag SAX-7S with sfGFP by CRISPR-Cas9, the only option was to insert the sfGFP very close to the predicted signal peptide of SAX-7S, which possibly affects the cleavage of the signal peptide or targeting of the protein. Thus, qv31 does not reliably inform about the subcellular localization of the protein SAX-7S, yet it yields valuable information about the spatio-temporal expression pattern of sax-7S.

\section{Domains Ig 3-4 of SAX-7S are necessary for its function in neuronal maintenance}

L1 family members play diverse roles via homophilic interactions through their extracellular domains which leads to homophilic cell adhesion (Brummendorf et al., 1998; Brummendorf and Rathjen, 1996; Haspel and Grumet, 2003; Hortsch, 2000), and mutating different extracellular Ig-like domains of vertebrate L1 perturbs its homophilic and/or heterophilic binding in in vitro assays (Blaess et al., 1998; Castellani et al., 2002; De Angelis et al., 1999; De Angelis et al., 2002; Felding-Habermann et al., 1997; Haspel et al., 2000; Holm et al., 1995; Kunz et al., 1998; Montgomery et al., 1996; Oleszewski et al., 1999; Zhao and Siu, 1995) and neurite outgrowth (Appel et al., 1993). In C. elegans, neuronal expression of a SAX-7S recombinant version lacking Ig5-6 domains rescued AIY/AVK neuronal soma position defects of sax-7(nj48) mutants, whereas a recombinant version lacking lg3-4 domains does not (Pocock et al., 2008). We asked whether such SAX-7S recombinant versions lacking specific Ig domains could rescue head ganglia organization in qv30 null mutant animals. We found that the transgene Punc-14::sax-7S $\Delta \lg 5-6$ rescued the position of the soma of the neurons ASH and ASI relative to the nerve ring in qv30 null mutants, but that the transgene Punc-14::sax-7S $\Delta$ lg3-4 did not rescue (Fig. 5C). This indicates that only lg domains 3 and 4 of SAX-7S are required for its role in the maintenance head ganglia organization.

Fnlll domains of L1 family members play diverse roles in neurite outgrowth, homophilic binding, and interactions with various partners (Haspel and Grumet, 2003; Holm et al., 1995; Kalus et al., 2003; Koticha et al., 2005; Maten et al., 2019; Silletti et al., 2000). We asked whether FnIII domains are necessary for sax-7S function in $C$. elegans to maintain head

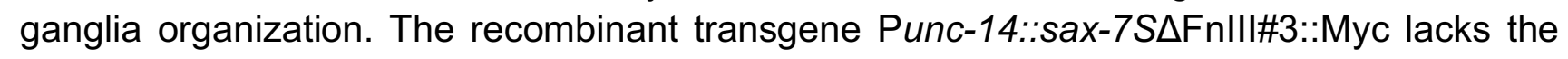
third Fnlll (Fnlll\#3) domain, which harbors the serine protease cleavage site (Fig. 5A, 
$\Delta$ Fnlll\#3). In extracts of $q v 30$ transgenic animals carrying this transgene, a $\sim 140 \mathrm{kDa}$ band is detected with anti-Myc antibodies (Fig. 5B), which is the expected size for uncleaved SAX$7 S$ minus the Fnlll\#3 domain (full-length SAX-7S would be $150 \mathrm{kDa}$ ). We found that this transgene rescues head ganglia organization defects of qv30 mutant animals (Fig. 5C), indicating that uncleaved SAX-7S $\triangle \mathrm{Fnlll} 33$ can function in neuronal maintenance, at least in a transgenic overexpression situation. We next tested a transgene which lacks all five FnIII domains, Punc-14::sax-7S $\triangle \mathrm{FnIII}$, and found that it also rescues the head ganglia organization defects of qv30 null mutants (Fig. 5C). Such transgene lacking all Fnlll domains could also rescue AIY and AVK soma position in nj48 mutants (Pocock et al., 2008), as well as AIY position and branching in sax-7(dz156) mutants (Diaz-Balzac et al., 2015).

The intracellular region (ICD) of SAX-7/L1CAM shows a strong homology between vertebrates and invertebrates, and mutations in the cytoplasmic domain leads to X-linked hydrocephalus in humans (Wong et al., 1995b). This intracellular part contains motifs (FERM, ankyrin and PDZ binding-domain motifs; Fig. 1B), which mediate interactions with intracellular components and cytoskeletal proteins (Davey et al., 2005; Davis and Bennett, 1994; Dirks et al., 2006; Falk et al., 2004; Gil et al., 2003; Gunn-Moore et al., 2006; Herron et al., 2009; Koroll et al., 2001; Schaefer et al., 2002; Wong et al., 1995a). We tested whether a transgene of sax-7S lacking the ankyrin-binding motif (Punc-14::sax-7S $\triangle$ ankyrin) could function to maintain head ganglia organization, and found that qv30 null mutants were significantly rescued by this transgene (Fig. 5C). Thus, the ankyrin-binding motif does not appear to be necessary for SAX-7S function in maintenance of head ganglia. We next asked if SAX-7S could function in neuronal maintenance without its intracellular domain (Punc14::sax-7S $\triangle I C D$ ), and found a partial but significant rescue of the qv30 defects in head ganglia organization in animals neuronally expressing this transgene (Fig. 5C). However, $40 \%$ of the qv30 animals display maintenance defects. This profound but incomplete rescue may be due to either mosaicism or overexpression of the plasmid, which could possibly interfere with interactions.

\section{Serine protease SAX-7S fragments can, together, function in neuronal maintenance}

400

The most abundant detected form of SAX-7 appears to be a serine protease-cleavage product (Fig. 1D), which splits the molecule within the third Fnlll domain (Fig. 1B). Other detected cleavage products result from a cleavage site proximal to the transmembrane domain (TM) (Fig. 1D). We tested whether the protein fragments predicted to result from serine proteasesite cleavage, or the TM-proximal cleavage, could function in maintaining neuronal organization, similarly to full-length SAX-7S. For this, we constructed four separate transgenes encoding each of the four predicted fragments of the protein SAX-7S, from cleavage at either the serine protease or the TM proximal sites. Some of these transgenes encode versions of SAX-7S with a C-terminal Myc tag, which can then be examined by immunoblots (Fig. 5A-B). Cleavage of SAX-7S at the serine protease site within the third FnIII domain results in $\mathrm{N}$ - and $\mathrm{C}$-terminal fragments, which we named "SAX-7S-fragment-A", and "SAX-7S-fragment-B", respectively (Fig. 5A). Another cleavage event proximal to the transmembrane domain results in $\mathrm{N}$ - and $\mathrm{C}$-terminal fragments which we named "SAX-7S413 fragment-C", and "SAX-7S-fragment-D", respectively (Fig. 5A). We tested each of these 414 fragments alone, or in reciprocal combinations, for their ability to rescue the neuronal 
415 maintenance defects of sax-7(qv30) mutants when expressed under the neuronal promoter Punc-14. Neither "SAX-7S-fragment-A" alone (Punc-14::sax-7S-[N-terminal] Ig3 up to serine 417 protease site), nor "SAX-7S-fragment-B alone" (Punc-14::sax-7S-serine protease site up to 418 PDZ [C-terminal]), could rescue head ganglia organization defects of qv30 mutant animals 419 (Fig. 5C). Similarly, neither "SAX-7S-fragment-C" alone (Punc-14::sax-7S-[N-terminal] Ig3 up 420 to TM site), nor "SAX-7S-fragment-D" alone (Punc-14::sax-7S-TM site up to PDZ [Cterminal]), could rescue head ganglia organization defects of $q v 30$ mutant animals (Fig. 5C).

We next tested whether the serine protease cleavage $\mathrm{N}$ - and C-terminal SAX-7S fragments together, i.e. "SAX-7S-fragment-A" and SAX-7S-fragment-B" together, or whether the TM-proximal cleavage $\mathrm{N}$ - and $\mathrm{C}$-terminal SAX-7S fragments together, i.e. "SAX-7Sfragment-C" and SAX-7S-fragment-D" together, could rescue neuronal maintenance defects of sax-7(qv30) mutant animals. To generate doubly transgenic animals harboring the two respective transgenes, we avoided simultaneously co-microinjecting the two transgenes, as DNA recombination events between two transgenes could potentially reconstitute a full-length gene. Thus, we instead used genetic crosses to generate doubly transgenic animals (harboring two independent extrachromosomal arrays). By genetic crosses between 2 different transgenic strains, we generated a doubly transgenic strain carrying the two extrachromosomal arrays for "SAX-7S-fragment-C" and "SAX-7S-fragment-D", which resulted in a strain with animals carrying both extrachromosomal arrays, used for rescue assays. We found that the combination of SAX-7S fragments $C$ and $D$ did not rescue sax7(qv30) mutant phenotype (Fig. 5C, "C+D"; two independent sets of extrachromosomal arrays were tested and failed to rescue). Thus, SAX-7S fragments $C$ and $D$ predicted to result from cleavage proximal to the TM domain, even when present simultaneously, cannot fulfill sax-7S function in neuronal maintenance.

In a similar manner, we crossed the strain carrying the extrachromosomal array that includes transgenic copies of "SAX-7S-fragment-A" with the second transgenic strain carrying the extrachromosomal array which includes transgenic copies of "SAX-7S-fragment-B". The resulting strain has animals carrying both extrachromosomal arrays, which we tested for rescue of $q v 30$ head ganglia organization defects. The simultaneous presence of both fragments $A$ and $B$, corresponding to the predicted products resulting from cleavage at the serine protease site, profoundly rescued the head ganglia organization in sax-7(qv30) mutant animals (Fig. 5C, "A+B"). This finding indicates that the two serine protease-cleavage products together can mediate neuronal maintenance. As an important control, we verified that the rescue observed in the doubly transgenic strain depends on the simultaneous presence of both transgenes (for fragments A and B). Indeed, each of the two re-derived in singly transgenic lines, each carrying only one of the two extrachromosomal arrays (fragment A alone: $n=28,82 \%$ defects, or fragment $B$ alone: $n=35,91 \%$ defects; at 1-day adult), no longer rescued the sax-7(qv30) defects, further confirming that only when the two SAX-7S fragments $A$ and $B$ are present together in an animal can then mediate neuronal maintenance (Fig. 5C, "A" and "B").

We analyzed protein extracts of doubly transgenic animals carrying both extrachromosomal arrays for fragments $A$ and $B$, and as expected, no full-length SAX-7S is detected (Fig. 5B), confirming that the distinct SAX-7S fragments $A$ and $B$, together, can fulfill the role of SAX-7S in neuronal maintenance. Together, our findings show that while the 
459 cleavage at the serine protease site is not absolutely necessary for SAX-7S function, at least 460 in an over-expression situation, the two cleaved fragments $A$ and $B$ resulting from it, 461 functionally complement to mediate normal SAX-7S function for the maintenance of neuronal 462 architecture in C. elegans. 


\section{DISCUSSION}

465

466

467

468

469

470

471

472

473

474

475

476

477

478

479

480

481

482

483

484

485

486

487

488

489

490

491

492

493

494

495

496

497

498

499

500

501

502

503

504

505

506

507

After initial establishment of the nervous system, neuronal maintenance molecules function to actively preserve neuronal structural organization and integrity. One such molecule is $C$. elegans SAX-7, homologous to vertebrate L1 proteins, whose developmental roles have been studied (Dong et al., 2013; Salzberg et al., 2013), but whose roles in the long-term maintenance of nervous system organization remain unclear. Here we have generated and characterized a complete loss-of-function allele of sax-7, examined the endogenous expression pattern of SAX-7S, tested the temporal requirements for sax-7S, and assessed the function of SAX-7S cleavage products in the maintenance of neuronal architecture.

\section{New sax-7 alleles: a complete null and two sax-7S-specific alleles}

The sax-7(nj48) allele, previously considered to be a null, has detectable sax-7 transcripts and proteins (Fig. 1). A new mutant, sax-7(qv30), deletes the entire sax-7 genomic locus, resulting in the complete loss-of-function of the gene (Figs. 1A,D). This null allele facilitates the interpretation of experiments without the caveat of potential truncated protein products present in hypomorphic alleles, which is especially important for rescue assays with transgenes encoding protein fragments.

qv30 mutant animals display defects that are in some cases stronger than previously studied alleles, for instance in the maintenance of some ganglia organization (e.g., AIY and AVK neuron pairs in the retrovesicular ganglion; PHA and PHB neuron pairs in the tail ganglion; Fig. 2). sax-7S(+), but not sax-7L(+), can rescue the defects of null mutants sax7(qv30) (Fig. 2A), supporting that sax-7S is the key isoform in maintenance of neuronal architecture, as previously described (Pocock et al., 2008; Sasakura et al., 2005). This is in accordance with previous reports that sax-7L-specific mutant alleles (eq2 and nj53) do not lead to neuronal maintenance defects (Benard et al., 2012; Pocock et al., 2008), and that sax$7 L(+)$ cannot rescue neuronal maintenance defects of mutants $n j 48$ and $k y 146$, where both sax-7 isoforms are affected (Pocock et al., 2008).

Our analysis of two new sax-7 short-specific alleles, qv25 and qv26, further supports the notion that sax-7S is the important isoform in neuronal maintenance. Mutant animals for each of these two sax-7S alleles display defects similar to the null qv30 (Fig. 2). As a note, other sax-7S-specific alleles with different molecular lesions have recently been isolated (Chen et al., 2019; Rahe et al., 2019). Together, our new findings and previous results unequivocally establish that SAX-7S is the important isoform mediating maintenance of neuronal architecture (Benard et al., 2012; Diaz-Balzac et al., 2015; Pocock et al., 2008; Sasakura et al., 2005; Wang et al., 2005).

\section{Post-embryonic expression of sax-7S is sufficient to maintain head ganglia organization}

Expression of sax-7S(+) during larval stages, which is well after the embryonic assembly of neuronal ganglia, is sufficient to function in maintaining ganglia organization (Fig. 3). Indeed, driving sax-7S expression (under the control of a heat shock promoter) at the $1^{\text {st }}$ larval stage, or as late as the $3^{\text {rd }}$ larval stage, was sufficient to profoundly rescue neuronal maintenance defects in qv30 null mutant animals. While the rescue is profound, it is not complete, possibly due to the mosaicism of the 
extrachromosomal array bearing the transgene and the failure to recapitulate normal sax-7S(+) expression levels. Nonetheless, larval expression profoundly rescues the null mutants, pointing to the fact that $\operatorname{sax}-7 S(+)$ functions post-developmentally to ensure the maintenance of neuronal organization. This finding rules out the possibility that the neuronal maintenance defects of sax-7 mutants are a result of an undetected embryonic defect that is amplified by growth and movement of the animal. Instead, our result is consistent with an active requirement for sax-7 post-embryonically to maintain the organization of an already established nervous system structure.

Thus far, only a handful of molecules have been identified that function to maintain specific aspects of the nervous system. This likely is a reflection of the difficulty associated with determining an adult role for molecules that also play critical roles during development. A post-embryonic neuronal role for sax-7, the $C$. elegans homologue of the mammalian L1CAM family, is a conserved property of this gene family. Indeed, loss of L1CAM specifically from the adult mouse brain led to an increase in basal excitatory synaptic transmission and behavioral alterations (Law et al., 2003). In rats, postdevelopmental nervous system knockdown of Neurofascin severely compromised the already established composition of the axon initial segment and led to an onset of motor deficits (Kriebel et al., 2011; Zonta et al., 2011). Postnatal disruption of CHL1 in excitatory neurons of the mouse forebrain affected the duration of working memory (Kolata et al., 2008). Thus, the continued importance of $L 1$ family members in the adult nervous system is conserved from worm to mammals, suggesting that our findings in $C$. elegans will likely have implications in other organisms.

\section{SAX-7S is robustly expressed across the nervous system}

Transgenic expression of sax-7S(+) under different tissue-specific promoters has been used to test for function (this study; (Benard et al., 2012; Diaz-Balzac et al., 2015; Dong et al., 2013; Pocock et al., 2008; Ramirez-Suarez et al., 2019; Salzberg et al., 2013; Sasakura et al., 2005; Zhou et al., 2008; Zhu et al., 2017). Here we have generated sfgfp insertion specifically in the sax-7S locus, and characterized its endogenous expression pattern. sfGFP::SAX-7S is robustly expressed in virtually all neurons (Fig. 4), consistent with the role of SAX-7S in the C. elegans nervous system. Indeed, transgenic wild-type copies of sax-7S(+) expressed panneuronally (Punc-14::sax-7S, Prab-3::sax-7S) rescue sax-7 mutant defects including head ganglia disorganization (Fig. 2A), PVQ axon flip-over, AlY and AVK neuronal soma displacement (Pocock et al., 2008), AIY soma position and branching (Diaz-Balzac et al., 2015), AFD neuronal soma position (Sasakura et al., 2005) and PVD length or defasiculation (Ramirez-Suarez et al., 2019), as well as neuronal SAX-7 expression rescues dendrite retrograde extension (Cebul et al., 2020). Interestingly, we observed that sfGFP.:SAX-7S expression levels vary among specific neurons in a given animal, and these neuron-specific differences appear to be reproducible across animals. Future studies will address the functional relevance of such SAX-7S expression level signatures.

Transgenic expression of sax-7S(+) in the hypodermis (using the epidermal promoter in Pdpy-7::sax-7(+) transgene), rescues the PVD dendrite defects of sax-7 mutants (Chen et al., 2019; Dong et al., 2013; Salzberg et al., 2013; Zhu et al., 2017). Despite our careful analyses of animals at all developmental stages, including with unmixing confocal microscopy, we did not observe sfGFP::SAX-7S expression in the body wall epidermis (hyp 7 cells). This suggests that either (1) the endogenous level of SAX-7S in the epidermis is too low to be detected, or (2) the functional form of SAX-7S, in this context, is the C-terminal 
serine protease cleavage product (fragment B), which cannot be seen with the qv31 sfGFP::SAX-7S knock-in, as the fluorescent protein is fused N-terminally (Fig. 5A). Consistent with this idea, PVD dendrites can be rescued with SAX-7S constructs lacking Nterminal domains Ig3-4 or Ig5-6 (Dong et al., 2013; Salzberg et al., 2013). Tagging the intracellular domain of SAX-7 may allow for visualization of epidermal expression, but such a construct cannot be specific to the short isoform (SAX-7S), if done at the endogenous genomic locus, as both isoforms share the entire intracellular C-terminal region. Previous immuno-histochemistry analyses using an antibody generated against the C-terminal cytoplasmic tail of SAX-7 reported expression of SAX-7 in multiple tissues, including robust signal in neuronal cell bodies, as well as in the nerve ring (major bundle of axons) and the ventral nerve cord (Chen et al., 2001; Wang et al., 2005).

\section{SAX-7S cleavage products in neuronal maintenance}

565

SAX-7S and SAX-7L proteins could be reliably distinguished on immunoblots thanks to robust controls: the mutant allele eq1, where the sequence coding for the intracellular domain of sax7 containing the epitope recognized by the antibody is deleted (Chen et al., 2001), and the null $q v 30$ where the entire sax-7 locus is deleted. We observed that in wild-type animals, (1) full-length SAX-7S is less abundant than the full-length SAX-7L; (2) the vast majority of SAX7 protein is cleaved, as an abundant $\sim 60 \mathrm{kDa}$ cleavage product, seemingly derived from the serine protease-cleavage site; and (3) another less abundant cleavage product of $\sim 28 \mathrm{kDa}$ may result from cleavage at a site near the transmembrane (Fig. 1D). In the sax-7S-specific alleles qv25 and qv26, where SAX-7S is absent, the abundance of full-length SAX-7L is similar to wild type, and the $\sim 60 \mathrm{kDa}$ serine protease cleavage product is less abundant compared to the wild-type, suggesting that the SAX-7S protein may be preferentially cleaved compared to SAX-7L. Also, in the sax-7L-specific alleles eq2 and nj53, the $\sim 60 \mathrm{kDa}$ serine protease-cleavage product appears more abundant than the wild type, perhaps revealing that the SAX-7S cleavage may be favored, resulting in a lower level of full-length SAX-7S versus full-length SAX-7L.

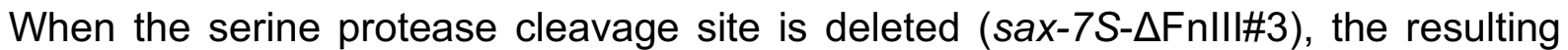
recombinant protein is functional in head ganglia maintenance (Fig. 5B,C), indicating that the cleavage is not essential for function in maintenance of neural architecture, at least with a highly expressed transgene. Consistent with this, motor neuron axon outgrowth defects upon knockdown of I1camb in zebrafish can be rescued by expression of a non-cleavable form of L1cam (Linneberg et al., 2019). However, this may be context-specific as Reelin-mediated cleavage of L1CAM in the mouse brain is important for neurodevelopment (Lutz et al., 2017). Furthermore, we find sax-7S- $\triangle \mathrm{FnIII \# 3} \mathrm{is} \mathrm{primarily} \mathrm{detected} \mathrm{as} \mathrm{full-length} \mathrm{via} \mathrm{western} \mathrm{blot} \mathrm{(Fig.}$ 5B), consistent with recent work which shows that mutating the cleavage site within the third FnIII domain of L1CAM leads to detectable full length protein with no Fnlll-domain mediated cleavage products detected (Kleene et al., 2020). This suggests that the third FnIII has conserved importance in SAX-7/L1CAM processing.

Although the two serine protease-cleavage products (SAX-7S-fragment-A and -B) cannot function individually in neuronal maintenance, we find that their simultaneous expression fulfills sax-7S neuronal maintenance function. The soluble ectodomain of L1cam similarly cannot solely restore $11 \mathrm{cam}$ knockdown-mediated defects in motor neuron axon 
596

597

598

599

600

601

602

603

604

605

606

607

608

609

610

611

612

613

614

615

616

617

618

619

620

621

622

623

624

625

626

627

628

629

630

631

632

633

634

635

636

637

638

639

outgrowth in zebrafish L1cam (Linneberg et al., 2019). It is possible that, in vivo, serine protease cleavage fragments $A$ and $B$ exist (as suggested by our immunoblot analysis) and may interact together to maintain neuronal architecture. In support of this, furin-mediated cleavage products of Tractin (the L1CAM homologue in leech) can interact in vitro, and these fragments together, not individually, can mediate adhesion in an in vitro S2 cell aggregation assay (Xu et al., 2003). As we know that SAX-7S can also promote homophilic adhesion in an in vitro cell aggregation assay (Sasakura et al., 2005), this points to the intriguing possibility that SAX-7S fragments together in vivo may have adhesive neural-maintenance-promoting properties. Future studies will help to address whether these SAX-7S fragments similarly function together or whether their function in neural maintenance is through other interacting factors.

\section{MATERIALS AND METHODS}

\section{Nematode strains and genetics}

Nematode cultures were maintained in an incubator at $20^{\circ} \mathrm{C}$ (unless otherwise noted) on NGM plates seeded with Escherichia coli OP50 bacteria as described (Brenner, 1974). Alleles used in this study are listed in Table 1. Strains were constructed using standard genetic procedures and are listed in Table 2. Genotypes were confirmed by genotyping PCR or by sequencing when needed. Primers used to build strains are listed in Table 3. All the mutant alleles and reporter strains are outcrossed with the Bristol N2 wild-type strain at least 3 times prior to use for analysis or strain building.

\section{RT-PCR for sax-7 alleles}

This analysis was performed with wild-type [N2], sax-7L-specific mutants [sax-7(eq2) and sax-7(nj53)], hypomorphic mutants of both isoforms [sax-7(nj48) and sax-7(tm1448)], and intracellular sax-7 mutant [sax-7(eq1)] strains. Total RNA was extracted from worm samples using Trizol (Invitrogen) according to manufacturer's instructions. RNA (500 ng) was reverse transcribed using the High Capacity cDNA Reverse Transcription Kit (Applied Biosystems) and random primers. PCR reactions were carried out with $1^{\text {st }}$ strand CDNA template, and 0.25 $\mu \mathrm{M}$ of each primer for sax-7 cDNA amplification in $10 \mathrm{mM}$ Tris $\mathrm{pH} 8.3,1.5 \mathrm{mM} \mathrm{MgCl}, 50 \mathrm{mM}$ $\mathrm{KCl}, 0.2 \mathrm{mM}$ deoxynucleotides, and $1 \mathrm{U}$ Phusion DNA polymerase for 30 cycles of $94^{\circ} \mathrm{C}$ for 10 seconds, $55^{\circ} \mathrm{C}$ for 20 seconds, and $72^{\circ} \mathrm{C}$ for 45 secs. Primers used to detect sax- 7 transcript are as following:

oCB985 (CGATTTGCAACTCAACAGGA),

oCB986 (TGGTGCTCATGAAGGATCAG),

oCB987 (GTGTCCCGAACTGATTCGAT),

oCB988 (TTTGTGGAACGTATTGACC),

oCB989 (GGAACGTATTGACCTGAAACAG),

oCB990 (TTGATCGTCCTGTCCGTGTA),

oCB991 (GACCACCGAATACCACAACC).

Primers oCB992 (TCGCTTCAAATCAGTTCAGC) and oCB993

(GCGAGCATTGAACAGTGAAG) were used for the control gene Y45F10D.4 (Hoogewijs et al., 2008) cDNA amplification. 
640

641

642

643

644

645

646

647

648

649

650

651

652

653

654

655

656

657

658

659

660

661

662

663

664

665

666

667

668

669

670

671

672

673

674

675

676

677

678

679

680

681

682

683

\section{Generation of sax-7 null allele by CRISPR-Cas9 (knockout)}

gRNA plasmids ( $p C B 392$ and $p$ CB393). The gRNAs plasmids were made as previously described (Arribere et al., 2014). To obtain a deletion of the entire locus of sax-7, we used two target sequences, one on the $1^{\text {st }}$ exon of the sax-7 long isoform (gtggccagtgagtaacaag reverse target sequence, pCB392) and the other one on the last exon of sax-7 corresponding to exon 17 and 14 of long and short isoform, respectively (ccggcatcaagctcttttg reverse target sequence, pCB393).

pCB392. Forward and reverse oligonucleotides (oCB1511: AAACcttgttactcactggccacC and oCB1510: TCTTGgtggccagtgagtaacaag, respectively), containing the 5 ' target sequence and overhangs compatible with Bsal sites in plasmid pRB1017 (Arribere et al., 2014), were annealed and ligated into pRB1017 cut with Bsal to create the gRNA plasmid pCB392.

pCB393. Forward and reverse oligonucleotides (oCB1513: AAACcaaaagagcttgatgccggC and oCB1512: TCTTGccggcatcaagctcttttg, respectively), containing the 3' target sequence and overhangs compatible with Bsal sites in plasmid pRB1017 (Arribere et al., 2014), were annealed and ligated into pRB1017 cut with Bsal to create the gRNA plasmid pCB393.

Plasmids were confirmed by sequencing with $\mathrm{M} 13$ reverse primer.

The repair donor ssDNA oligonucleotide (repair template). We designed the repair donor simple-strand DNA oligonucleotide and ordered to Integrated DNA Technologies (IDT) (oCB1514:

GATTCTAGATCACGTCGAAAGACCACCATCATGAGGAGCTTCATATTTCTAGCTTGATG CCGGCCGAACGGCCCGAGAAAGGATCAACGTCGACGTTTG, forward). The donor sequence starts with 50 nucleotides corresponding to $5^{\prime}$ homology arm of sax-7L at the 5' target site, followed by 49 nucleotides corresponding to 3' homology arm of sax-7 at the 3' target site.

sax-7 deletion is located from $8373 \mathrm{bp}$ to $28330 \mathrm{bp}$ on cosmid C18F3, deletion of $19957 \mathrm{bp}$ (Table 1, Fig. S1A).

Microinjection. DNA mixture was prepared in injection buffer $(20 \mathrm{mM}$ potassium phosphate, $3 \mathrm{mM}$ potassium citrate, 2\% PEG, pH 7.5). The injection mix contained the Cas9 plasmid (pDD162; (Dickinson et al., 2013) at $50 \mathrm{ng} / \mu \mathrm{L}$, the gRNA plasmids pCB392 and pCB393 at $50 \mathrm{ng} / \mu \mathrm{L}$ each, the ssDNA donor oCB1514 at $20 \mathrm{ng} / \mu \mathrm{L}$, the gRNA plasmid pJA58 (dpy-10 target; (Arribere et al., 2014) at $50 \mathrm{ng} / \mu \mathrm{L}$ and the ssDNA repair template for $d p y-10$ (dpy-10(cn64); (Arribere et al., 2014) at $20 \mathrm{ng} / \mu \mathrm{L}$. Mutations in the $d p y-10$ gene were used as CRISPR co-conversion marker.

Screening. F1 progeny were screened for Rol and Dpy phenotypes 3-4 days after injection. Rol or Dpy F1 animals were singled and the F2 progeny were screened by PCR for the absence of sax-7 gene with 2 couples of primers. First couple of primers outside sax-7, oCB747 (TCTCTCAAAATTCTTCGCAAGC, forward) and oCB1025 (CGGGAAGAAATGAAACAGGA, reverse), giving a band when sax-7 is knockout works. Second couple of primers inside sax-7, oCB212 (GAAATACACACAAATACGAGTGC, forward) and oCB723 (TAGTTGATTAAAATGTTTCAAGATTG, reverse) giving a band in wild type (no knockout of sax-7).

Identification. The strain resulting from this genome editing is identified as sax-7(qv30) (Tables 1-3) and verified by sequencing the deletion junctions (Fig. S1A) and also failed to amplify any product by several PCR reactions with primers targeting most of sax-7 exons. 
684

685

686

687

688

689

690

691

692

693

694

695

696

697

698

699

700

701

702

703

704

705

706

707

708

709

710

711

712

713

714

715

716

717

718

719

720

721

722

723

724

725

726

727

\section{Generation of sax-7(qv25) and sax-7(qv26), sax-7S-specific alleles by CRISPR-Cas9} Two insertion-deletion mutants, namely sax-7(qv25) and sax-7(qv26) (Tables 1-3, Fig. S1BC), were obtained during our efforts to insert sfgfp in the sax-7S-specific locus by CRIPSRCas9, described below.

\section{Generation sfGFP::SAX-7S by CRISPR-Cas9 (knock-in)}

We chose the protein marker sfGFP as a gene tag because it encodes a GFP variant that folds robustly even when fused to poorly folded proteins and its modified structure resists to the acidic extracellular environment (Pedelacq et al., 2006).

gRNA plasmids ( $p C B 394$ and $p C B 395$ ). The gRNAs plasmids were made as previously described (Arribere et al., 2014). Two target sequences were selected at the end of the exon 1 of sax-7S-specific locus (sax-7S/C18F3.2a,d), located in the predicted sax-7S signal peptide (ggatgtctactgttccttg forward target sequence (pCB394) and tgaaatgaaactaaccaca reverse target sequence ( $p C B 395)$ ).

pCB394. Forward and reverse oligonucleotides (oCB1515: TCTTGggatgtctactgttccttg and oCB1516: AAACcaaggaacagtagacatccC, respectively), containing the target sequence and overhangs compatible with Bsal sites in plasmid pRB1017 (Arribere et al., 2014), were annealed and ligated into pRB1017 cut with Bsal to create the gRNA plasmid pCB394.

pCB395. Forward and reverse oligonucleotides (oCB1518: AAACtgtggttagttcatttcaC and oCB1517: TCTTGtgaaatgaaactaaccaca, respectively), containing the target sequence and overhangs compatible with Bsal sites in plasmid pRB1017 (Arribere et al., 2014), were annealed and ligated into pRB1017 cut with Bsal to create the gRNA plasmid pCB395.

Plasmids were confirmed by sequencing with $\mathrm{M} 13$ reverse primer.

The repair donor PCR amplicon (repair template). We decided to design the repair donor DNA in order that the new gene insertion take place directly at the end of the exon 1 of sax-7S, in sax-7S signal peptide. The end of the sax-7S signal peptide is at beginning of the exon 2 of sax-7S. Thus, it was necessary to add this signal sequence part localized downstream the insertion area (TCGGATCGCTACTACACA at the beginning of exon 2) at the end of exon 1, along with the gene sfgfp to be inserted, so as to ensure the presence of the entire signal peptide (Figs. 4B, S1D).

The repair donor DNA was amplified by PCR using first, primers oCB1525 (GTGTCGGATCGCTACTACACAATGAGCAAAGGAGAAGAAC, forward) and oCB1527 (ATGTGCCCTAAAAAGAAAAATGAAATGAAACTAACTTTGTAGAGCTCATCCATGC, reverse) and a plasmid containing the sequence of sfGFP as template. Primers oCB1525 contains 18 bases in 5' upstream sfgfp corresponding to the missing sax-7S signal peptide sequence part and oCB1527 contains 35 bases corresponding to 3' homology arms of sax$7 S$ at the target site. A second PCR was amplified on the previous products with primers oCB1526

(TCATATTCCTGCTAGGATGTCTACTGTTCCTTGTGTCGGATCGCTAC, forward) and

oCB1527 (ATGTGCCCTAAAAAGAAAAATGAAATGAAACTAACTTTGTAGAGCTCATCCATGC, reverse). Primer oCB1526 contains 35 bases corresponding to 5 ' homology arms of sax-7S at the target site. sfgfp with signal peptide part were inserted immediately following amino acid 29 of SAX-7S. 
Microinjection. DNA mixture was prepared in injection buffer $(20 \mathrm{mM}$ potassium phosphate, $3 \mathrm{mM}$ potassium citrate, 2\% PEG, pH 7.5). The injection mix contained the Cas9 plasmid (pDD162; (Dickinson et al., 2013) at $50 \mathrm{ng} / \mu \mathrm{L}$, the gRNA plasmids pCB394 and pCB395 at $25 \mathrm{ng} / \mu \mathrm{L}$ each, the 5'arm::sp::sfgfp::3'arm donor PCR (containing the signal peptide, $s p$ ) at $100 \mathrm{ng} / \mu \mathrm{L}$, the gRNA plasmid pJA58 (dpy-10 target; (Arribere et al., 2014) at $50 \mathrm{ng} / \mu \mathrm{L}$ and the ssDNA repair template for $d p y-10$ (dpy-10(cn64); (Arribere et al., 2014) at $20 \mathrm{ng} / \mu \mathrm{L}$. Mutations in the $d p y-10$ gene were used as CRISPR co-conversion marker.

Screening. F1 progeny were screened for Rol and Dpy phenotypes 3-4 days after injection. Rol or Dpy F1 animals were singled and the F2 progeny were screened by PCR for the presence of sax-7S signal peptide and sfgfp in the sax-7S locus with primers oCB1022 (TGGTGGTAGCGATGGTGTAG, forward) and oCB818 in sfgfp (TTCAGCACGCGTCTTGTAGG, reverse) for the 5' insertion side and oCB1427 in sfgfp (AAAAGCGTGACCACATGGTCC, forward) and oCB1023 (AGTTCGATGTTCTCGGCTGT, reverse) for the 3 ' insertion side.

Identification. The new strain resulting from this genome editing is identified as sax7(qv31[sfgfp::sax-7S]) (Tables 1, 2), which is abbreviated as sfgfp::sax-7S. The modified locus was verified by sequencing of the entire region (Fig. S1D).

\section{Microinjection to generate transgenic animals}

747

DNA constructs are described in the Molecular Cloning section. Briefly, for sax-7 constructs, the sax-7 cDNA was subcloned under the control of pan-neuronal promoters rab-3 (Nonet et al., 1997) and unc-14 (Ogura et al., 1997) or heat shock promoter hsp-16.2 that express in neurons and other tissues (Fire et al., 1990; Jones et al., 1986; Stringham et al., 1992). Transgenic animals were generated by standard microinjection techniques (Mello and Fire, 1995). Each construct was injected at $1 \mathrm{ng} / \mu \mathrm{L}$ (pCB191), $5 \mathrm{ng} / \mu \mathrm{L}$ (pCB219, pCB213, pCB402 and pCB212), $10 \mathrm{ng} / \mu \mathrm{L}$ (pCB224 and pCB426), or $25 \mathrm{ng} / \mu \mathrm{L}$ (pCB428, pCB189, pCB195, pCB430, pCB429, pCB431, pCB401 and pCB432), along with one or two co-injection markers to select transgenics, including Pceh-22::gfp (50 $\mathrm{ng} / \mu \mathrm{L})$ and Plgc-11::gfp (50 $\mathrm{ng} / \mu \mathrm{L})$ labelling the pharynx in green, Pttx-3::mCherry (50 ng/ $\mu \mathrm{L})$ labelling AIY neurons in red, and Punc122::rfp (50 $\mathrm{ng} / \mu \mathrm{L})$ labelling coelomocytes in red. When needed, pBSK+ was used to increase total DNA concentration of the injection mixes to $200 \mathrm{ng} / \mu \mathrm{L}$. For details on transgenic strains and their injection mix composition, see Table 2.

760

\section{Molecular cloning}

762

763

764

765

The gene coding sequences of sax-7/C18F3.2b and sax-7/C18F3.2a were used for the long and short isoform respectively (available on WormBase). All inserts were verified by sequencing.

\section{Construct to express SAX-7S post-developmentally}

767

768

Phsp16.2::sax-7S (pCB191). Vector pRP100 (Punc-14::sax-7S; (Pocock et al., 2008)) was digested with HindIII and BamHI to release Punc-14 and ligated with insert of Phsp-16.2 digested out of pPD49.78 (was a gift from Andrew Fire; Addgene plasmid \# 1447; 
Constructs to express variants of SAX-7 under pan-neuronal promoters

Prab-3::sax-7S (pCB428). Used for rescue experiments (Gift from H.E. Bülow (Ramirez-Suarez et al., 2019)).

Punc-14::sax-7S::Myc (pCB189). Used for rescue experiments and western blot against Myc. Cloned by Gibson assembly. For this plasmid we used the vector pRP100 (Punc-14::sax-7S; (Pocock et al., 2008)). The FLAG::sax-7S::Myc construct was made by amplifying the 5' end of the sax-7S cDNA from pRP100, carrying a BamHI site, with two nested PCR reactions adding FLAG tag sequence (GATTACAAGGATGACGACGATAAG) right after the signal peptide sequence in the exon 2 and, by amplifying the 3' end of sax-7S cDNA from pRP100, carrying Ncol site, with two nested PCR reactions adding Myc tag sequence (GAGCAGAAACTCATCTCTGAAGAGGATCTG) right before the stop codon, in the exon 14. The vector pRP100 was digested with BamHI and Ncol enzymes to release nontagged sax-7S cDNA in order to clone the synthesized fragment FLAG::sax-7S::Myc into it with the same restriction enzymes. As a note, western blot experiments with several antiFLAG antibodies were done in the attempt of detecting the N-terminus part of SAX-7, but failed.

Punc-14::sax-7L (pCB195). Used for rescue experiments. Cloned through Gibson assembly. The HA::SAX-7L::V5 construct was made by amplifying the 5' end of the sax-7L cDNA from Punc-17::sax-7L construct, carrying BamHI site, with two nested PCR reactions adding HA tag sequence (TACCCATACGACGTCCCAGACTACGCT) after the signal peptide sequence (exon 1) in the exon 2 (between 60-61 sax-7L cDNA bases). Also, by amplifying the 3' end of sax-7L cDNA carrying Ncol site, with two nested PCR reactions adding $V 5$ tag sequence (GGTAAGCCTATCCCTAACCCTCTCCTCGGTCTCGATTCTACG) right before the stop codon, in the exon 17. The vector pRP100 was digested with BamHI and Ncol enzymes to release non tagged sax-7S cDNA in order to clone the synthesized fragment HA::SAX-7L::V5 into it with the same restriction enzymes.

Punc-14::sax-7S $\triangle$ lg3-4 (pCB430). Used for rescue experiments (Gift from H.E. Bülow, (Pocock et al., 2008)).

Punc-14::sax-7S $\triangle$ Ig5-6 (pCB429). Used for rescue experiments (Gift from H.E. Bülow, (Pocock et al., 2008)).

Punc-14::sax-7S $\triangle$ FnIII\#3 (pCB224). Used for rescue experiments (Pocock et al., 2008).

Punc-14::sax-7S $\triangle$ Fnlll\#3::Myc (pCB426). Used for rescue experiments and western blot against Myc. Vector pCB189 (Punc-14::FLAG::sax-7S::Myc) was digested with Pstl and Sall restriction enzymes to release the sax-7S cDNA fragment containing the Fnlll\#3 domain and ligated with insert of sax-7S cDNA fragment without the Fnlll\#3 domain, digested out of pCB224 (Punc-14::sax-7S $\triangle F n I I I \# 3$; (Pocock et al., 2008)) with the same restriction enzymes. 
815 As a note, western blot experiments with several anti-FLAG antibodies were done in the attempt of detecting the N-terminus part of SAX-7, but failed.

Punc-14::sax-7S $\Delta$ FnIII (pCB431). Used for rescue experiments (Gift from H.E. Bülow (Diaz-Balzac et al., 2015)).

Punc-14::sax-7S $\triangle$ Ankyrin (pCB401). Used for rescue experiments. The vector Pttx-

Punc-14::sax-7S $\triangle I C D$ (pCB432). Used for rescue experiments (Gift from H.E. Bülow (Ramirez-Suarez et al., 2019)).

Punc-14::sax-7S Ig3 to serine protease cleavage site (RWKR) (Fragment A) (pCB219). Used for rescue experiments and western blot against Myc. From pCB189 (Punc14::FLAG::sax-7S::Myc), the sax-7S cDNA fragment FLAG::Ig3 to serine protease cleavage site (RWKR) (amino acid 745) was amplified with primers oCB798 (CATGATgctagcATGGGGTTACGAGAGACGATGG, forward) and oCB799 (ATCATGccatggCTATCTCTTCCATCTGAACTTTC, reverse) to add on Nhel and Ncol restriction sites, respectively. Vector pCB195 (Punc-14::HA::sax-7L::V5) was digested with Nhel and Ncol and ligated with the insert of sax-7S cDNA fragment using the same restriction enzymes. As a note, western blot experiments with several anti-FLAG antibodies were done in the attempt of detecting the N-terminus part of SAX-7, but failed.

Punc-14::sax-7S serine protease cleavage site (RWKR) to PDZ::Myc (Fragment B) (pCB213). Used for rescue experiments and western blot against Myc. In this case, we needed to be careful adding a signal peptide sequence to assess an accurate expression of the variant. Thus, from pCB189 (Punc-14::FLAG::sax-7S::Myc), the sax-7S cDNA fragment serine protease cleavage site (RWKR) (amino acid 742) to PDZ::Myc was amplified with primers oCB811 (ACTGGCCACATATCATCAGGCAGCATAGATTGGTCAGCGAGATGGAAGAGATCAATTC G, forward) and oCB801 (ATCATGccatggCTACAGATCCTCTTCAGAGATG, reverse) to add the sax-7L signal peptide sequence and an Ncol restriction site, respectively. This first nest product was then amplified with primers (CATGATgctagcATGAGGAGCTTCATATTCCTCTTGTTACTCACTGGCCACATATCATCAG $G$, forward) and oCB801 (ATCATGccatggCTACAGATCCTCTTCAG

AGATG, reverse) to add Nhel restriction site. Then, vector pCB195 (Punc-14::HA::sax$7 L:: V 5)$ was digested with Nhel and Ncol and ligated with the insert of sax-7S cDNA fragment using the same restriction enzymes.

Punc-14::sax-7S Ig3 to proximal-transmembrane cleavage site (Fragment C) (pCB402). Used for rescue experiments. From pCB189 (Punc-14::FLAG::sax-7S::Myc), the sax-7S cDNA fragment lg3 to proximal-transmembrane cleavage site (amino acid 1024) was 
amplified with primers oCB798 (CATGATgctagcATGGGGTTACGAGAGACGATGG, forward) and oCB807 (ATCATGccatggCTAACGAGAACTCGTTCCCGTCG, reverse) to add Nhel and Ncol restriction sites, respectively. Then, vector pCB195 (Punc-14::HA::sax-7L::V5) was digested with Nhel and $\mathrm{Ncol}$ and ligated with the insert of sax-7S cDNA fragment using the same restriction enzymes.

864

Punc-14::sax-7S proximal-transmembrane cleavage site to PDZ::Myc (Fragment D) (pCB212). Used for rescue experiments. We needed to be careful adding a signal peptide sequence to assess an accurate expression of the variant. From pCB189 (Punc14::FLAG::sax-7S::Myc), the sax-7S cDNA fragment from proximal-transmembrane cleavage site (amino acid 1024) to PDZ::Myc was amplified with primers oCB813 (TCACTGGCCACATATCATCAGGCAGCATAGATTGGTCAGCGGAAAGAAATGTCTATCT TTTG, forward) and oCB801 (ATCATGccatggCTACAGATCCTCTT

873 CAGAGATG, reverse) to add the sax-7L signal peptide sequence and Ncol restriction site, respectively. This first nested product was then amplified with primers oCB812 (CATGATgctagcATGAGGAGCTTCATATTCCTCTTGTTACTCACTGGCCACATATCATCAG G, forward) and oCB801 (ATCATGccatggCTACAGATCCTCTTCAG 876 AGATG, reverse) to add on Nhel restriction site. Then, vector pCB195 (Punc-14::HA::sax$7 L:: V 5)$ was digested with Nhel and Ncol and ligated with the insert of sax-7S cDNA fragment using the same restriction enzymes.

\section{Protein analysis of endogenous SAX-7 levels in sax-7 mutant alleles}

881

882

This analysis was performed with wild-type [oyls14], sax-7S-specific mutants [sax-7(qv25); oyls14 and sax-7(qv26); oyls14], sax-7L-specific mutants [sax-7(eq2); oyls14 and sax7(nj53); oyls14], null mutant [sax-7(qv30); oyls14], hypomorphic mutant of both isoforms [sax7(nj48); oyls14], and intracellular sax-7 mutant for antibody specificity control [sax-7(eq1); oyls 14] strains. Worms were fed and grown on plates at $20^{\circ} \mathrm{C}$ for at least three generations before collecting.

For each strain, either (a) 100 L4-stage animals were collected in M9 solution and bacteria was washed off, or (b) large populations of worms were collected. Because the amount of SAX-7 protein was too low to detect all the protein forms on the analysis above, large pellets of thousands of mixed-stage worm populations were collected by washing plates, mostly devoid of bacteria, with M9 solution.

NETI (NaCl, EDTA, Tris, IGEPAL) buffer and protease inhibitors (Roche 892 \#11836153001) were added to worm pellets with 2X Laemmli sample buffer (Bio-Rad \#1610737 ) and $5 \% \beta$-mercaptoethanol $(\mathrm{v} / \mathrm{v})$, and immediately frozen in liquid nitrogen. Samples were boiled for $5 \mathrm{~min}$ at $95^{\circ} \mathrm{C}$ and centrifuged for $10 \mathrm{~min}$ at $10000 \mathrm{rpm}$ prior to loading with

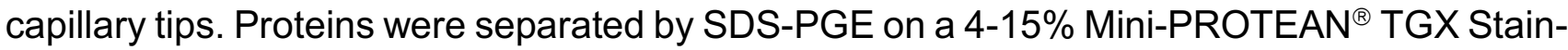
Free $^{\mathrm{TM}}$ gel (Bio-Rad \#456-8084) and transferred with the Trans-Blot ${ }^{\circledR}$ Turbo $^{\mathrm{TM}}$ RTA Transfer Kit (Bio-Rad \#170-4275) to a LF (low fluorescence) PVDF membrane using the Trans-Blot ${ }^{\circledR}$ Turbo $^{\mathrm{TM}}$ Transfer System (Bio-Rad). Membranes were blocked in 5\% BSA (VWR \#0175), 5\% non-fat milk and incubated in 1:8000 rabbit anti-SAX-7, an affinity purified antibody generated against the SAX-7 cytoplasmic tail [gift of (Chen et al., 2001)] and 1:5000 goat anti-rabbit HRP secondary antibody (Bio-Rad \#170-5046). For the loading control, membranes were 
903 incubated in 1:1000 rabbit anti-HSP90 antibody (CST \#4874) and 1:5000 goat anti-rabbit 904 HRP secondary antibody (Bio-Rad \#170-5046). Signal was revealed using Clarity Max ${ }^{\mathrm{TM}}$ 905 Western ECL Substrate (Bio-Rad \#170-5062), and imaged using the ChemiDoc ${ }^{\text {TM }}$ System 906 907 (Bio-Rad). This analysis was performed three times for each set of experiments.

\section{Protein analysis of transgenic SAX-7S protein fragments}

Myc tag was used (see sax-7S transgenes section). This analysis was performed with wildtype [hdls29] and qv30 null mutant transgenic animals carrying different sax-7S protein fragments under the pan-neuronal promoter Punc-14: sax-7S "Fragment A" [Ig3 up to serine protease cleavage site] (VQ1059), "Fragment B" [serine protease cleavage site up to Cterminal::Myc] (VQ1062), sax-7S-A and -B fragments together [lg3 up to serine protease cleavage site and serine protease cleavage site up to C-terminal::Myc] (VQ1065), sax-7S full length [sax-7S::Myc] (VQ1357), and sax-7S without serine protease cleavage site in the $3^{\text {rd }}$ Fnlll [sax-7SAFnlll\#3::Myc] (VQ1449).

For each strain, before collecting, worms were allowed to grow for $\sim 2$ generations by feeding with $\sim 30$ transgenic worms (or non-transgenic for wild type). Because these assays require large pellets of thousands of worms, rather than picking transgenic animals, worms were collected by washing populations on plates. We estimate that around $\sim 50 \%$ of animals carry the various extrachromosomal transgenes (described above). Indeed, unstable nonintegrated extrachromosomal arrays are lost during cell divisions and over generations, so that by the time that the worms were collected from plates, not all, but a proportion of the animals on the plates are transgenics (this was verified by visual inspection). Mixed-stage worm populations from plates devoid of bacteria were collected in M9 solution. Then, NETI ( $\mathrm{NaCl}$, EDTA, Tris, IGEPAL) buffer and protease inhibitors (Roche \#11836153001) were add to worm pellets with 2X Laemmli sample buffer (Bio-Rad \#161-0737), 5\% $\beta$-mercaptoethanol $(\mathrm{v} / \mathrm{v})$, and immediately frozen in liquid nitrogen. Each sample provided enough material to load 2 gel wells allowing the visualization of SAX-7S recombinants tagged by Myc. Samples were boiled for $5 \mathrm{~min}$ at $95^{\circ} \mathrm{C}$ and centrifuged for $10 \mathrm{~min}$ at $10000 \mathrm{rpm}$ prior to loading with capillary tips, separated by SDS-PGE on a $4-15 \%$ Mini-PROTEAN ${ }^{\circledR}$ TGX Stain-Free ${ }^{\mathrm{TM}}$ gel (Bio-Rad \#456-8084), and transferred with the Trans-Blot ${ }^{\circledR}$ Turbo $^{\text {TM }}$ RTA Transfer Kit (BioRad \#170-4275) to a LF (low fluorescence) PVDF membrane using the Trans-Blot $^{\circledR}$ Turbo $^{\mathrm{TM}}$ Transfer System (Bio-Rad). Membranes were blocked in 5\% BSA (VWR \#0175), 5\% non-fat milk. Blots were incubated in 1:500 mouse anti-Myc (CST \#2276) and 1:3000 goat anti-mouse HRP secondary antibody (Jackson ImmunoResearch \#115-035-003). For the loading control, membranes were incubated in 1:1000 rabbit anti-HSP90 antibody (CST \#4874) and 1:5000 goat anti-rabbit HRP secondary antibody (Bio-Rad \#170-5046). Signal was revealed using Clarity Max ${ }^{\mathrm{TM}}$ Western ECL Substrate (Bio-Rad \#170-5062), and imaged using the ChemiDoc $^{\mathrm{TM}}$ System (Bio-Rad). This analysis was performed three times.

\section{Microscopy and imaging}

Worms were grown in incubator at $20^{\circ} \mathrm{C}$ for at least 3 generations prior to analysis. Worm stages are indicated in the figures. $24 \mathrm{~h}$ post-L4 stage is considered " ${ }^{\text {st }}$ day of adulthood", $24 \mathrm{~h}$ after that is considered "day 2 of adulthood", and so on. 


\section{Neuroanatomical observations}

948 Neuroanatomy was examined in wild-type and mutant animals using specific reporters. 949 Worms were anesthetized with $75 \mathrm{mM}$ of sodium azide $\left(\mathrm{NaN}_{3}\right)$ and mounted on $5 \%$ agarose 950 pads on glass slides. Animals were observed with Nomarski or fluorescence microscopy (Carl 951 Zeiss Axio Scope.A1 or Axio Imager.M2), and images were acquired using the AxioCam 952 camera (Zeiss) and processed using AxioVision (Zeiss), with 60x oil immersion objective (expected for PVQ/PVP axons: 100x oil immersion objective).

Analysis of ASH/ASI cell body positioning with respect to the nerve ring. Cell body pairs of $\mathrm{ASH} / \mathrm{ASI}$ chemosensory neurons and the nerve ring (neuropil of the worm), positioned in the head ganglia of the worm, were visualized using hdls29 (Schmitz et al., 2008), an integrated Psra-6::DsRed2; Podr-2::cfp reporter as well as oyls14 (Sarafi-Reinach et al., 2001), an integrated Psra-6::gfp reporter. Animals were analyzed in a lateral orientation. Normally, both the two ASH and the two ASI soma are located posterior to the nerve ring. Animals were counted as mutant when at least one ASH or ASI soma was touching, on top of, or anterior to the nerve ring. Animals were counted as wild type when all ASH/ASI soma were positioned posterior to the nerve ring.

964

Analysis of AVKJAIY soma. Cell body pairs of AVK/AIY interneurons, were visualized using a stock containing two integrated reporters, bwls2 (Pflp-1::gfp) to label AVK in green, and otls133 (Pttx-3::rfp) to label AIY in red (Pocock et al., 2008). Animals were analyzed when in a ventral orientation. Cell bodies of AVK/AIY localized to the head ganglia of the worm in the retrovesicular ganglion. Normally, both neuron pairs AVKL/AIYL (left) and AVKR/AIYR (right) adhere to each other (Pocock et al., 2008; White et al., 1986b). Animals were counted as mutant when one or two AVK/AIY pairs were detached. Animals were counted as wild type when both of the AVK/AIY soma pairs were in contact.

Analysis of PHA/PHB soma. Cell body pairs of PHA/PHB chemosensory neurons, were visualized using Dil (1,1'-Dioctadecyl-3,3,3',3'-Tetramethylindocarbocyanine Perchlorate) staining procedure (Hedgecock et al., 1985). This is a lipophilic fluorescent stain for labeling cell membranes and hydrophobic structures, providing an alternative for labeling cells and tissues. In our case, it allows us to stain and visualize by a pink fluorescence the ciliated amphid (ADL, ASH, ASI, ASJ, ASK, AWB) and phasmid (PHA, PHB) neurons (Collet et al., 1998), that are exposed to the outside environment. Animals were analyzed in a ventral orientation. Cell bodies of PHA/PHB localized to the tail ganglia of the worm in the lumbar ganglion. Normally, both neuron pairs PHAL/PHBL (left) and PHAR/PHBR (right) adhere to each other (White et al., 1986a). Animals were counted as mutant when any of the PHA/PHB pairs were detached from one another. Animals were counted as wild type when both of the PHA/PHB soma pairs were in contact.

986

Analysis of $P V Q / P V P$ axons. PVQ and PVP axons were visualized in animals using $h d l s 29$ (Schmitz et al., 2008), an integrated Psra-6::DsRed2; Podr-2::cfp reporter, labelling PVQ and 
991 fascicle of the ventral nerve cord, respectively. Animals were counted as having an axon flip-

992 over defect when one of the PVQ/PVP axons was flipped to the opposite fascicle at any point

993 along the ventral nerve cord, as previously described (Benard et al., 2006).

994

Other phenotypic observations

Analysis of embryonic lethality. From plates with hermaphrodites laying eggs, a lot of embryos were picked with OP50, and spread into a new plate that was kept at $20^{\circ} \mathrm{C}$. After $\sim 16 \mathrm{hr}$, the number of larvae and dead embryos were counted. This experiment was repeated 3 times. Analysis of brood size. An L4 worm was singled on a new plate independently. The number of embryos laid were counted each day of adulthood until 4-days-old adults and the total amount of laid embryos during 4 days was calculated. This was done at least 7 times. Analysis of egg-laying. Ten L4-stage worms were put on one plate and their ability to lay embryos normally was examined each day from day 1 to 5 of adulthood. Worms deficient in embryo laying retain them inside their bodies and display an Egl phenotype (Desai and Horvitz, 1989; Trent et al., 1983). When counted defective they were removed from the plate. This was done 10 times.

1006

\section{Expression pattern analysis of sfGFP::SAX-7S}

1009 Fluorescence images of sax-7::ty1::egfp::3FLAG strain (Fig. 4A; (Sarov et al., 2012)) were 1010 captured by fluorescence microscopy (Carl Zeiss Axio Imager.M2), and images were 1011 acquired using the AxioCam camera (Zeiss) and processed using AxioVision (Zeiss), with 60x oil immersion objective.

Fluorescent images of $q v 31$, the sfgfp::sax-7S strain (Fig. 4B), were captured using a Nikon A1R confocal microscope and processed using ImageJ. For each stage, at least 8 worms were examined in detail. Nematodes were immobilized in $75 \mathrm{mM}$ of $\mathrm{NaN}_{3}$ and mounted on $5 \%$ agarose pads on glass slides. All fluorescence images for sfgfp::sax-7S strain were obtained with the same settings using a Nikon Ti-e spinning disk confocal with 60x oil immersion objective. Images were three-dimensionally unmixed with NIS-Elements image acquisition and analysis software. Green fluorescent background is commonly seen in worms (gut granules), which disturbs the analysis of green fluorescent fusion proteins. In this study, we took advantage of a microscopy technique which "unmixes" overlapping spectral emissions after acquisition. Thanks to highly sensitive GaAsP-detectors, signals can be distinguished by the process called "spectral unmixing" (Ackermann, 2017).

For this, we acquired images for wild type N2 animals and determined a ROI in the pharynx in the head of the worm, giving a spectral profile defined as "background" green autofluorescence the worm. Then, with the sfgfp::sax-7S CRISPR-Cas9 strain, which expresses "real" green fluorescence, we acquired images and determined a ROI to the soma part of one neuron in the head of the worm, giving a spectral profile defined as "real" green fluorescence in the case of the sfGFP fluorophore. Finally, the "background" profile was subtracted from the "real" green fluorescence profile, keeping the real green fluorescence emission coming from sfGFP for the entire animal. ND2 files generated with NIS-Elements were imported into Fiji for analysis. Maximum intensity projections were generated by selecting stacks that had both ventral and dorsal signals. 


\section{Heat-shock inducible expression of sax-7S(+)}

1037

This analysis was performed with wild type [oyls14], null mutant [sax-7(qv30); oyls14] and null mutant transgenic animals carrying sax-7S cDNA under heat shock promoter $h s p 16.2$ that express in neurons and other tissues (Fire et al., 1990; Jones et al., 1986; Stringham et al., 1992). Worms were maintained in the incubator at $15^{\circ} \mathrm{C}$ for at least two generations prior to analysis. To generate freshly hatched pools of L1s, plates were fed with a lot of adult hermaphrodites (which carry eggs) and left at $15^{\circ} \mathrm{C}$ around $15 \mathrm{~h}$ (overnight) in order to have many laid embryos close to hatch. Then, embryos were picked on a new plate and kept for $\sim 6 \mathrm{~h}$ at $15^{\circ} \mathrm{C}$, after which any remaining unhatched embryos were removed from the plates leaving only freshly hatched L1s (on average $3.5 \mathrm{~h}$ old) on the plate. Animals were either heat shocked immediately as freshly L1s, or as L3s ( $42 \mathrm{~h}$ post-hatch). Heat shock treatment consisted of 3 cycles of 30 minutes at $37^{\circ} \mathrm{C}$ with a 60 minutes recovery period at $20^{\circ} \mathrm{C}$ between each cycle, after which plates were put back at $15^{\circ} \mathrm{C}$ until analysis as adults (Fig. 3A). All experiments were repeated at least twice. Neuroanatomical analysis of ASH/ASI cell body positioning with respect to the nerve ring (see Neuroanatomical observations) were performed on animals as 1-, 2-, 3-, 4-, and 5-days-old adults.

1052

\section{Quantification and statistical analysis}

1054

z-tests and student's t test were performed in MS Office Excel. Error bars in bar graphs represent standard error of proportion (S.E.P.).

\section{Data availability}

Mutant and genome engineered strains will be available at the Caenorhabditis Genetics Center, and all strains and plasmids are available upon request. The authors affirm that all data necessary for confirming the conclusions of the article are present within the article, figures, and tables.

\section{ACKNOWLEDGMENTS}

1064

1065 We thank Maria Doitsidou and Lise Rivollet for comments on the manuscript; Denis Flipo for assistance with confocal microscopy and unmixing; Andrea Thackeray and Lise Rivollet for help throughout the project; the following researchers for sharing reagents: Max Heiman (for a plasmid containing the sfgfp gene); Lihsia Chen (for anti-C-terminal SAX-7 antibodies); Roger Pocock and Hannes Bülow for plasmids, as well as the CGC, which is funded by the NIH Office of Research Infrastructure Programs (P40 OD010440), and WormBase. This work was supported by funds from the NIH, CIHR, NSERC, and FRQS to C.Y.B.; and scholarships to V.E.D. by UQÀM and the CERMO-FC Research Center. 
1075 Table 1. List of sax-7 mutant alleles used.

\begin{tabular}{|c|c|c|c|}
\hline Allele & Nature of alleles & $\begin{array}{l}\text { Location on } \\
\text { cosmid C18F3 }\end{array}$ & Reference \\
\hline$q v 31$ & $\begin{array}{l}732 \text { bp insertion } \\
\text { sfGFP::SAX-7S construct }\end{array}$ & after 12809 & This study \\
\hline$q v 30$ & $\begin{array}{l}19,972 \text { bp deletion } \\
\text { Total loss of function }\end{array}$ & 8364-28335 & This study \\
\hline$q v 25$ & $\begin{array}{l}47 \text { bp insertion, which } \\
\text { creates an ORF frameshift and a stop } \\
\text { codon in sax-7S signal peptide }\end{array}$ & after 12785 & This study \\
\hline$q v 26$ & $\begin{array}{l}36 \text { bp insertion } \\
\text { In frame but disrupts sax-7S signal } \\
\text { peptide }\end{array}$ & after 12785 & This study \\
\hline eq2 & 648 bp deletion & $8041-8688$ & (Wang et al., 2005) \\
\hline nj53 & 724 bp deletion & $8122-8845$ & (Sasakura et al., 2005) \\
\hline nj48 & 582 bp deletion & $12457-13038$ & (Sasakura et al., 2005) \\
\hline $\operatorname{tm} 1448$ & 1,727 bp deletion & $22599-24325$ & $\begin{array}{l}\text { Mitani lab at NBRP } \\
\text { C. elegans }\end{array}$ \\
\hline eq1 & 2,020 bp deletion & $26591-28605$ & (Wang et al., 2005) \\
\hline
\end{tabular}


1079 Table 2. List of strains used.

\begin{tabular}{|c|c|c|}
\hline Name & Transgene & Reference \\
\hline \multicolumn{3}{|c|}{ Wild-type reference strains } \\
\hline \multirow[t]{2}{*}{ N2 } & & (Brenner, 1974) \\
\hline & $\begin{array}{l}\text { Psra-6::DsRed2; } \\
\text { Podr-2::cfp }\end{array}$ & (Schmitz et al., 2008) \\
\hline $\mathrm{OH} 4589$ & Pflp-1::gfp, Pttx-3:::fp & (Pocock et al., 2008) \\
\hline VQ51 & Psra-6::gfp & (Sarafi-Reinach et al., 2001) \\
\hline \multicolumn{3}{|c|}{ sax-7S knock-ins } \\
\hline VQ1290 & sax-7(qv31) IV & This study \\
\hline TH502 & $\begin{array}{lc}\text { unc-119(ed3) } & \text { III; }[\text { sax- } \\
\text { ddls290 } & 7:: \text { ty1::egfp::3FLAG] } \\
\end{array}$ & (Sarov et al., 2012) \\
\hline \multicolumn{3}{|c|}{ sax-7 mutants } \\
\hline VQ1047 & sax-7(qv30) IV & This study \\
\hline VQ1058 & $\begin{array}{l}\text { sax-7(qv30) IV; hdls29 } \\
V\end{array}$ & This study \\
\hline VQ1057 & sax-7(qv30) IV; bwls2 otls133 II & This study \\
\hline VQ1000 & $\begin{array}{l}\text { sax-7(qv30) IV; oyls14 } \\
V\end{array}$ & This study \\
\hline $\mathrm{OH} 4587$ & sax-7(nj48) IV & (Sasakura et al., 2005) \\
\hline OH7984 & sax-7(nj48) IV; oyls14 V & (Pocock et al., 2008) \\
\hline VQ397 & sax-7(nj48) IV; hdls29 V & This study \\
\hline $\mathrm{OH} 4588$ & sax-7(nj48) IV; bwls2 ot/s133 II & (Pocock et al., 2008) \\
\hline VQ976 & sax-7(qv25) IV & This study \\
\hline VQ1011 & $\begin{array}{l}\text { sax-7(qv25) IV; oyls14 } \\
V\end{array}$ & This study \\
\hline VQ1269 & $\begin{array}{l}\text { sax-7(qv25) IV; hdls29 } \\
V\end{array}$ & This study \\
\hline VQ1259 & $\begin{array}{l}\text { sax-7(qv25) IV; } \\
\text { bwls2 otls133 II }\end{array}$ & This study \\
\hline VQ977 & sax-7(qv26) IV & This study \\
\hline VQ1012 & $\begin{array}{l}\text { sax-7(qv26) IV; oyls14 } \\
V\end{array}$ & This study \\
\hline $\mathrm{LH} 2$ & sax-7(eq2) IV & (Wang et al., 2005) \\
\hline $\mathrm{OH} 6028$ & sax-7(eq2) IV; oyls14 V & (Benard et al., 2012) \\
\hline LH81 & sax-7(eq1) IV & (Wang et al., 2005) \\
\hline $\mathrm{OH} 8904$ & sax-7(eq1) IV; oyls14 V & (Benard et al., 2012) \\
\hline IK637 & sax-7(nj53) IV & (Sasakura et al., 2005) \\
\hline $\mathrm{OH} 9002$ & sax-7(nj53) IV; oyls14 V & (Benard et al., 2012) \\
\hline & sax-7(tm1448) IV & $\begin{array}{l}\text { Mitani lab at NBRP C. } \\
\text { elegans; (Wang et al., 2005)) }\end{array}$ \\
\hline
\end{tabular}




\begin{tabular}{|c|c|c|c|}
\hline VQ1357 & $\begin{array}{l}\text { sax-7(qv30) IV; hdls29 } \\
\text { V; qvEx377 }\end{array}$ & $\begin{array}{l}\text { pCB189, Plgc-11::gfp, } \\
\text { pBSK+. Line \#1 }\end{array}$ & This study \\
\hline VQ1358 & $\begin{array}{l}\text { sax-7(qv30) IV; hdls29 } \\
\text { V; qvEx378 }\end{array}$ & $\begin{array}{l}\text { pCB189, Plgc-11::gfp, } \\
\text { pBSK+. Line \#2 }\end{array}$ & This study \\
\hline VQ1359 & $\begin{array}{l}\text { sax-7(qv30) IV; hdls29 } \\
\text { V; qvEx379 }\end{array}$ & $\begin{array}{l}\text { pCB189, P/gc-11::gfp, } \\
\text { pBSK+. Line \#3 }\end{array}$ & This study \\
\hline VQ1566 & $\begin{array}{l}\text { sax-7(qv30) IV; hdls29 } \\
\text { V; qvEx476 }\end{array}$ & $\begin{array}{l}\text { pCB428, Punc-122::rfp, } \\
\text { pBSK+, Line \#1 }\end{array}$ & This study \\
\hline VQ1587 & $\begin{array}{l}\text { sax-7(qv30) IV; hdls29 } \\
\text { V; qvEx485 }\end{array}$ & $\begin{array}{l}\text { pCB428, Punc-122::rfp, } \\
\text { pBSK+, Line \#2 }\end{array}$ & This study \\
\hline VQ1465 & $\begin{array}{l}\text { sax-7(qv30) IV; oyls14 } \\
\text { V; qvEx234 }\end{array}$ & $\begin{array}{l}\text { pCB191, Punc-122::rfp, } \\
\text { Pttx-3::mCherry, } \\
\text { pBSK+ }\end{array}$ & This study \\
\hline VQ1375 & $\begin{array}{l}\text { sax-7(qv30) IV; hdls29 } \\
\text { V; qvEx391 }\end{array}$ & $\begin{array}{l}\text { pCB195, Plgc-11::gfp, } \\
\text { pBSK+. Line \#1 }\end{array}$ & This study \\
\hline VQ1377 & $\begin{array}{l}\text { sax-7(qv30) IV; hdls29 } \\
\text { V; qvEx393 }\end{array}$ & $\begin{array}{l}\text { pCB195, Plgc-11::gfp, } \\
\text { pBSK+. Line \#2 }\end{array}$ & This study \\
\hline VQ1583 & $\begin{array}{l}\text { sax-7(qv30) IV; hdls29 } \\
\text { V; qvEx481 }\end{array}$ & $\begin{array}{l}\text { pCB430, Punc-122::rfp, } \\
\text { pBSK+. Line \#1 }\end{array}$ & This study \\
\hline VQ1588 & $\begin{array}{l}\text { sax-7(qv30) IV; hdls29 } \\
\text { V; qvEx486 }\end{array}$ & $\begin{array}{l}\text { pCB430, Punc-122::rfp, } \\
\text { pBSK+. Line \#2 }\end{array}$ & This study \\
\hline VQ1590 & $\begin{array}{l}\text { sax-7(qv30) IV; hdls29 } \\
\text { V; qvEx488 }\end{array}$ & $\begin{array}{l}\text { pCB430, Punc-122::rfp, } \\
\text { pBSK+. Line \#3 }\end{array}$ & This study \\
\hline VQ1584 & $\begin{array}{l}\text { sax-7(qv30) IV; hdls29 } \\
\text { V; qvEx482 }\end{array}$ & $\begin{array}{l}\text { pCB429, Punc-122::rfp, } \\
\text { pBSK+. Line \#1 }\end{array}$ & This study \\
\hline VQ1586 & $\begin{array}{l}\text { sax-7(qv30) IV; hdls29 } \\
\text { V; qvEx484 }\end{array}$ & $\begin{array}{l}\text { pCB429, Punc-122::rfp, } \\
\text { pBSK+. Line \#2 }\end{array}$ & This study \\
\hline VQ1589 & $\begin{array}{l}\text { sax-7(qv30) IV; hdls29 } \\
\text { V; qvEx487 }\end{array}$ & $\begin{array}{l}\text { pCB429, Punc-122::rfp, } \\
\text { pBSK+. Line \#3 }\end{array}$ & This study \\
\hline VQ1449 & $\begin{array}{l}\text { sax-7(qv30) IV; hdls29 } \\
\text { V; qvEx441 }\end{array}$ & $\begin{array}{l}\text { pCB426, Punc-122::rfp, } \\
\text { Pttx-3::mCherry, } \\
\text { pBSK+. Line \#1 }\end{array}$ & This study \\
\hline VQ1116 & $\begin{array}{l}\text { sax-7(qv30) IV; oyls14 } \\
\text { V; qvEx309 }\end{array}$ & $\begin{array}{l}\text { pCB224, Punc-122::rfp, } \\
\text { Pttx-3::mCherry, } \\
\text { pBSK+. Line \#2 }\end{array}$ & This study \\
\hline VQ1117 & $\begin{array}{l}\text { sax-7(qv30) IV; oyls14 } \\
\text { V; qvEx310 }\end{array}$ & $\begin{array}{l}\text { pCB224, Punc-122::rfp, } \\
\text { Pttx-3::mCherry, } \\
\text { pBSK+. Line \#3 }\end{array}$ & This study \\
\hline VQ1582 & $\begin{array}{l}\text { sax-7(qv30) IV; hdls29 } \\
\text { V; qvEx480 }\end{array}$ & $\begin{array}{l}\text { pCB431, Punc-122::rfp, } \\
\text { pBSK+. Line \#1 }\end{array}$ & This study \\
\hline VQ1594 & $\begin{array}{l}\text { sax-7(qv30) IV; oyls14 } \\
\text { V; qvEx489 }\end{array}$ & $\begin{array}{l}\text { pCB431, Punc-122::rfp, } \\
\text { pBSK+. Line \#2 }\end{array}$ & This study \\
\hline VQ1112 & $\begin{array}{l}\text { sax-7(qv30) IV; oyls14 } \\
\text { V; qvEx305 }\end{array}$ & $\begin{array}{l}\text { pCB401, Punc-122::rfp, } \\
\text { Pttx-3::mCherry, } \\
\text { pBSK+. Line \#1 }\end{array}$ & This study \\
\hline VQ1113 & $\begin{array}{l}\text { sax-7(qv30) IV; oyls14 } \\
\text { V; qvEx306 }\end{array}$ & $\begin{array}{l}\text { pCB401, Punc-122::rfp, } \\
\text { Pttx-3::mCherry, } \\
\text { pBSK+. Line \#2 }\end{array}$ & This study \\
\hline
\end{tabular}




\begin{tabular}{|c|c|c|c|}
\hline VQ1114 & $\begin{array}{l}\text { sax-7(qv30) IV; oyls14 } \\
\text { V; qvEx307 }\end{array}$ & $\begin{array}{l}\text { pCB401, Punc-122::rfp, } \\
\text { Pttx-3::mCherry, } \\
\text { pBSK+. Line \#3 }\end{array}$ & This study \\
\hline VQ1585 & $\begin{array}{l}\text { sax-7(qv30) IV; hdls29 } \\
\text { V; qvEx483 }\end{array}$ & $\begin{array}{l}\text { pCB432, Punc-122::rfp, } \\
\text { pBSK+. Line \#1 }\end{array}$ & This study \\
\hline VQ1596 & $\begin{array}{l}\text { sax-7(qv30) IV; hdls29 } \\
\text { V; qvEx490 }\end{array}$ & $\begin{array}{l}\text { pCB432, Plgc-11::gfp, } \\
\text { pBSK+. Line \#2 }\end{array}$ & This study \\
\hline VQ1597 & $\begin{array}{l}\text { sax-7(qv30) IV; hdls29 } \\
\text { V; qvEx491 }\end{array}$ & $\begin{array}{l}\text { pCB432, Plgc-11::gfp, } \\
\text { pBSK+. Line \#3 }\end{array}$ & This study \\
\hline VQ1059 & $\begin{array}{l}\text { sax-7(qv30) IV; hdls29 } \\
\text { V; qvEx243 }\end{array}$ & $\begin{array}{l}\text { pCB219, Pceh-22::gfp, } \\
\text { pBSK+ }\end{array}$ & This study \\
\hline VQ1062 & $\begin{array}{l}\text { sax-7(qv30) IV; hdls29 } \\
\text { V; qvEx246 }\end{array}$ & $\begin{array}{l}\text { pCB213, Punc-122::rfp, } \\
\text { pBSK+ }\end{array}$ & This study \\
\hline VQ1118 & $\begin{array}{l}\text { sax-7(qv30) IV; hdls29 } \\
\text { V; qvEx311 }\end{array}$ & $\begin{array}{l}\text { pCB402, Pceh-22::gfp, } \\
\text { pBSK+. Line \#1 }\end{array}$ & This study \\
\hline VQ1119 & $\begin{array}{l}\text { sax-7(qv30) IV; hdls29 } \\
\text { V; qvEx312 }\end{array}$ & $\begin{array}{l}\text { pCB402, Pceh-22::gfp, } \\
\text { pBSK+. Line \#2 }\end{array}$ & This study \\
\hline VQ1121 & $\begin{array}{l}\text { sax-7(qv30) IV; hdls29 } \\
\text { V; qvEx314 }\end{array}$ & $\begin{array}{l}\text { pCB212, Punc-122::rfp, } \\
\text { pBSK+. Line \#1 }\end{array}$ & This study \\
\hline VQ1120 & $\begin{array}{l}\text { sax-7(qv30) IV; hdls29 } \\
\text { V; qvEx313 }\end{array}$ & $\begin{array}{l}\text { pCB212, Punc-122::rfp, } \\
\text { pBSK+. Line \#2 }\end{array}$ & This study \\
\hline VQ1065 & $\begin{array}{l}\text { sax-7(qv30) IV; hdls29 } \\
\text { V; qvEx243; qvEx246 }\end{array}$ & $\begin{array}{l}\text { pCB219, Pceh-22::gfp, } \\
\text { pBSK+ and pCB213, } \\
\text { Punc-122::rfp, pBSK+ }\end{array}$ & This study \\
\hline VQ1123 & $\begin{array}{l}\text { sax-7(qv30) IV; hdls29 } \\
\text { V; qvEx311; qvEx314 }\end{array}$ & $\begin{array}{l}\text { pCB402, Pceh-22::gfp, } \\
\text { pBSK+. Line \#1 and } \\
\text { pCB212, Punc-122::rfp, } \\
\text { pBSK+. Line \#1 }\end{array}$ & This study \\
\hline VQ1122 & $\begin{array}{l}\text { sax-7(qv30) IV; hdls29 } \\
\text { V; qvEx312; qvEx313 }\end{array}$ & $\begin{array}{l}\text { pCB402, Pceh-22::gfp, } \\
\text { pBSK+. Line \#2 and } \\
\text { pCB212, Punc-122::rfp, } \\
\text { pBSK+. Line \#2 }\end{array}$ & This study \\
\hline VQ1129 & $\begin{array}{l}\text { sax-7(qv30) IV; hdls29 } \\
\text { V; qvEx311; qvEx246 }\end{array}$ & $\begin{array}{l}\text { pCB402, Pceh-22::gfp, } \\
\text { pBSK+. Line \#1 and } \\
\text { pCB213, Punc- } \\
\text { 122::rfp, pBSK+ }\end{array}$ & This study \\
\hline
\end{tabular}


1083 Table 3. List of primers used to genotype the gene sax-7 when build strains.

\begin{tabular}{|c|c|c|c|c|}
\hline Allele & Primer & Sequence & $\begin{array}{l}\text { PCR } \\
\text { product(s) } \\
\text { (bp) }\end{array}$ & Cosmid coordinates \\
\hline \multirow{6}{*}{$q v 30$} & Mutant specific & & & \\
\hline & oCB747 & tctctcaaaattcttcgcaagc & 326 & C18F3 8252...8273, forward \\
\hline & oCB1025 & cgggaagaaatgaaacagga & & C18F3 $28531 \ldots 28550$, reverse \\
\hline & Wild-type specific & & & \\
\hline & oCB1022 & tggtggtagcgatggtgtag & 609 & C18F3 12312...12331, forward \\
\hline & oCB1023 & agttcgatgttctcggctgt & & C18F3 12901...12920, reverse \\
\hline \multirow{2}{*}{$q v 25$} & oCB1022 & tggtggtagcgatggtgtag & $656(\mathrm{mt})$ & C18F3 12312...12331, forward \\
\hline & oCB1023 & agttcgatgttctcggctgt & 609 (wt) & C18F3 12901...12920, reverse \\
\hline \multirow{2}{*}{$q v 26$} & oCB1022 & tggtggtagcgatggtgtag & $645(\mathrm{mt})$ & C18F3 12312...12331, forward \\
\hline & oCB1023 & agttcgatgttctcggctgt & 609 (wt) & C18F3 12901...12920, reverse \\
\hline \multirow{2}{*}{ nj48 } & oCB1022 & tggtggtagcgatggtgtag & $257(\mathrm{mt})$ & C18F3 $12312 \ldots 12331$, forward \\
\hline & оCB208 & gagttattggggtattttagcg & 825 (wt) & C18F3 13115...13136, reverse \\
\hline
\end{tabular}


1096

1097

1098

1099

1100

1101

1102

1103

1104

1105

1106

1107

1108

1109

1110

1111

1112

1113

1114

1115

1116

1117

1118

1119

1120

1121

1122

1123

1124

1125

1126

1127

1128

1129

1130

1131

1132

1133

1134

1135

1136

1137

1138

\section{FIGURE LEGENDS}

\section{Fig. 1. Analysis of sax-7 mutant alleles.}

(A) Schematics of the gene structure for the sax-7 short (C18F3.2a) and long (C18F3.2b) isoforms. The mutant alleles used in this study are indicated, including the newly generated the null allele $q v 30$ and sax-7S-specific alleles $q v 25$ and $q v 26$ (see Fig. S1 A-C for sequence information). Alleles $n j 48, t m 1448$, and eq1 affect both isoforms, and alleles eq2 and $n j 53$ are sax-7L-specific (see Table 1 for allele information).

(B) Schematics of the protein structure of SAX-7L and SAX-7S. Red arrows indicate cleavage sites: serine protease cleavage site in Fnlll\#3, or cleavage site proximal to the transmembrane (TM) domain. The two N-terminal Ig domains $\lg 1$ and $\lg 2$ may fold at the hinge region onto Ig3 and Ig4, indicated in grey (Pocock et al., 2008).

(C) Schematics of the four encoded sax-7 isoforms. Isoforms a and d, and isoforms b and c, are nearly identical, except for a short sequence of 9 extra nucleotides at the beginning of exons 17 and 14 in isoforms $c$ and d, respectively. sax-7 mutant alleles and primers used for RT-PCR analysis are indicated. Primer oCB990 (blue) was used to detect the long isoforms ( $b$ and $c$ ). Primer oCB991 (green) was used to detect the short isoforms (a and d). Primer oCB989§ (brown) specifically targets isoforms $\mathrm{c}$ and d, as its 3' end sequence primes on the 9 extra nucleotides of isoforms $c$ and $d$. Conversely, primer oCB988* (violet) specifically targets isoforms $a$ and $b$, as it was designed to prime at the exon junction lacking the 9 extra nucleotides. To the right, detection of sax-7 transcripts by RT-PCR. All RT-PCR products were confirmed by sequencing, and correspond to the predicted sax-7 sequences. In mutant $\underline{n j 48}$, transcripts are detected. The sax-7 long isoforms (b and c) RT-PCR product amplified with the primers oCB990/oCB987 is shorter than in the wild type, in accordance with the nj48 deletion where exon 5 of sax-7L is deleted. As expected, (primer oCB991 falls within the nj48 deletion), no transcript for short isoforms (a and d) were detected. In mutants ni53 and eq2, the 5' UTR and exon 1 of the sax-7 long isoforms (b and c) are deleted, and for eq2, part of exon 2 is deleted as well. Yet, sax-7 long (b and c) transcripts are detected in nj53 and eq2. Finally, in mutants tm1448 and eq1, both long (b and $c$ ) and short (a and d) transcripts are detected. Y45F10D.4 is a housekeeping gene used as an RT-PCR control (Hoogewijs et al., 2008).

(D) Western blot analyses of SAX-7 protein. An antibody specific to the intracellular domain (ICD) of SAX-7 was used, which detects a region in the C-terminus of SAX-7S and SAX-7L (Chen et al., 2001). anti-HSP90 was used as a loading control. "+" indicates wild-type strain. Representative membranes of at least 3 independent repeats each. Asterisks $\left(^{*}\right)$ denote nonspecific bands, which are unrelated to SAX-7 as they are present in extracts of: (1) sax7(qv30) null mutants, where the entire sax-7 genomic locus is deleted, and (2) eq1 mutant, where the entire the region coding for the epitope targeted by this antibody is deleted. "?" indicates an unknown form of SAX-7, which is detected in both wild type and sax-7 mutants, except for the null $q v 30$ and the epitope-control eq1.

Left panel: $>5000$ mixed-stages worm populations were loaded per well. The band corresponding to full-length SAX-7L $(\sim 190 \mathrm{kDa})$ is indicated by a blue arrow; SAX-7L is detected in the wild type and in mutants qv25 and qv26, but not in eq2 and $n j 53$, as expected. 
1139 The band corresponding to full-length SAX-7S $(\sim 150 \mathrm{kDa})$ is indicated by a green arrow; SAX-

$11407 \mathrm{~S}$ is detected in the wild type and in mutants eq2 and nj53, but not in qv25 and qv26, as

1141 expected. A presumably truncated mutant version of SAX-7L is detected in eq2 (blue

1142 arrowhead), which is not detected in wild type or eq1 and qv30 controls. Also, a truncated

1143 mutant version of SAX-7 is detected in nj48 (black arrowhead, unclear whether it corresponds

1144 to a truncated SAX-7S and/or SAX-7L).

1145 Two cleavage products are also detected: a highly abundant band at $\sim 60 \mathrm{kDa}$, indicated by

1146 a red arrow, corresponds to the C-terminal product resulting from cleavage at the serine

1147 protease site; a less abundant band at $\sim 28 \mathrm{kDa}$, indicated by a black arrow, corresponds to

1148 the C-terminal product from the cleavage site near to the transmembrane domain, and

1149 appears to run as a double band. An exposure of $9.5 \mathrm{sec}$ is required to see the bands of full-

1150 length SAX-7L and SAX-7S (arrows); however, at this exposure, the $\sim 60 \mathrm{kDa}$ cleavage

1151 product saturates the area indicated by the red dotted box. To better distinguish level

1152 differences among mutants, the same $\sim 60 \mathrm{kDa}$ membrane area was exposed for $0.1 \mathrm{sec}$ and

1153 is shown underneath. In SAX-7S-specific mutants qv25 and qv26, the $60 \mathrm{kDa}$ C-terminal

1154 product (resulting from cleavage at the serine protease site) is detected at a lower level of

1155 compared to wild type; however, in SAX-7L-specific mutants eq2 and nj53 the level of this 60

$1156 \mathrm{kDa}$ C-terminal serine protease cleavage products is comparable to wild type, suggesting that

1157 most of this cleavage product may be derived from full-length SAX-7S. On the other hand,

1158 the $28 \mathrm{kDa}$ C-terminal cleavage (resulting product from cleavage site near to TM) appears to

1159 be less abundant in eq2.

1160 Right panel: 100 L4 worms (4 ${ }^{\text {th }}$ larval stage) were loaded per well. While not all protein forms

1161 can be detected with this lower protein amount, the $60 \mathrm{kDa}$ C-terminal product from cleavage

1162 at the serine protease site is again clearly detected at lower levels in the sax-7S-specific

1163 mutants qv25 and qv26, compared wild type.

Fig. 2. Neuronal maintenance defects in the sax-7 mutant alleles qv30, qv25 and qv26.

1166 (A) sax-7S is required to maintain head ganglia organization post-developmentally. (A')

1167 Fluorescence images of the head region, where the soma and axons of the chemosensory

1168 neurons $\mathrm{ASH}$ and $\mathrm{ASI}$ are visualized using reporter Psra-6::DsRed2. Drawings illustrate

1169 microscopy images. Reporters Psra-6::DsRed2 (hdls29) and Psra-6::gfp (oyls14) give

1170 comparable results for all genotypes tested. In the wild type, the soma of neurons ASH/ASI

1171 (red arrowheads) are positioned posteriorly relative to the nerve ring (yellow arrowhead)

1172 throughout stages. In sax-7 mutants, the relative positioning between the soma of neurons

$1173 \mathrm{ASH} / \mathrm{ASI}$ and the nerve ring is initially normal (soma posterior to nerve ring), but becomes

1174 progressively defective in late larvae onwards (soma can either overlap with or become

1175 anterior to the nerve ring). (A") Quantification of the relative positioning between the ASH/ASI

1176 soma and the nerve ring in wild type, null mutant qv30, and sax-7S-specific mutants qv25 and

$1177 q v 26$. Animals were examined at the $2^{\text {nd }}(\mathrm{L} 2)$ and $4^{\text {th }}(\mathrm{L} 4)$ larval stages, as well as at days 1 ,

11782 , or 5 of adulthood. Rescue of qv30 null mutant defects by expression of sax-7S(+) in the

1179 nervous system using the heterologous promoters Punc-14 and Prab-3 (expression of sax-

$11807 L(+)$ does not rescue). Relative positioning between the soma of neurons ASH/ASI and the

1181 nerve ring was examined at 1-day adulthood using reporter Psra-6::DsRed2. Statistical

1182 comparisons are with qv30 mutant. 
1183 (B) sax-7S is required to maintain the retrovesicular ganglion organization. (B') Fluorescence 1184 images showing the soma of two pairs of interneurons AVK and AIY on either sides of the 1185 animal, visualized using reporters Pflp-1::gfp and Pttx-3::DsRed2. In the wild type animals, 1186 the soma of AVK (green) and AIY (red/yellow when overlap) are adjacent with each other. In 1187 sax-7 mutants, one or both of the AVK and AIY neuron pairs become separate from one 1188 another. (B") Quantification of animals showing separate pairs of AVK and AIY soma in wild 1189 type, null mutant qv30, sax-7S-specific mutant $q v 25$, and hypomorphic mutant $n j 48$, at the $4^{\text {th }}$ 1190 larval stage (L4) and days 1 and 2 of adulthood. The qv30 null and qv25 sax-7S-specific 1191 mutants are more affected than nj48 mutants.

1192 (C) sax-7S functions to maintain tail ganglia organization. (C') Fluorescence images of the 1193 chemosensory neurons PHA and PHB, visualized using Dil staining, whose soma are located 1194 in the lumbar ganglia on each side of the animal. In the wild type, the PHA and PHB soma 1195 are adjacent to each other. In sax-7 mutants, one or both of the PHA/PHB pairs are separated 1196 from one another. (C") Quantification of disorganized soma position in wild-type, null mutant $1197 q v 30$, sax-7S-specific mutant qv25, and hypomorphic mutant $n j 48$, at the $4^{\text {th }}$ larval stage. The qv30 null and qv25 sax-7S-specific mutants are more severe than nj48 mutants. Scale bar, $10 \mu \mathrm{m}$. Sample size is indicated under each column of the graph. Error bars are standard error of the proportion. Asterisks denote significant difference: * $p \leq 0.05,{ }^{* *} p \leq 0.01$, ${ }^{* * *} p \leq 0.001$. (z-tests, $p$ values were corrected by multiplying by the number of comparisons, Bonferroni correction). "+" indicates wild-type strain; n.s., not significant.

Fig. 3. Expression of sax-7S(+) during larval stages is sufficient for sax-7S to function in the maintenance of neuronal organization.

1206 (A) Summary of the heat shock experiments performed. Animals were kept at $15^{\circ} \mathrm{C}$ at all 1207 times except during heat shock at $37^{\circ} \mathrm{C}$ (red boxes). Heat shock was done at either the $1^{\text {st }}$ 1208 (L1) or the $3^{\text {rd }}(\mathrm{L} 3)$ larval stage. Animals were later analyzed at days $1,2,3,4$, and 5 of 1209 adulthood.

1210 (B) Quantification of the relative position between the soma of ASH/ASI and the nerve ring (as in Fig. 2A), visualized using the reporter oyls14 (Psra-6::gfp), at days 1, 2, 3, 4 and 5 of adulthood (age indicated under each bar of the graph). Transgenic animals carry a transgene of sax-7S(+) expressed under the control of a heat-shock promoter (Phsp-16.2::sax-7S(+)). Controls include the wild type, sax-7(qv30) mutants, and non-transgenic siblings of the transgenic animals, which are derived from the same mothers and grew on the same plates, but which do not carry the extrachromosomal array harboring the transgene. Additionally, for all of the four genetic conditions, neuroanatomical analyses were done in the absence of heat shock so as to ensure that no transgene expression occurred in the absence of heat shock. The defects that adult sax-7(qv30) mutants normally display are profoundly rescued by heatshock-induced expression of sax-7S(+) at larval stages, as seen in heat-shocked adult sax7(qv30) mutants carrying the transgene, (orange bars). Non-transgenic siblings, however, are severely defective, indicating that the rescue of defects is dependent on expression of sax$7 S(+)$ upon heat shock.

"+", indicates wild-type; "NO HS", no heat-shock; "HS L1", heat shock was performed at the $1^{\text {st }}$ larval stage; "HS L3", heat shock was performed at the $3^{\text {rd }}$ larval stage. Sample sizes is indicated along the grey zone, under each bar of the graph. Error bars are standard error of 
1227 the proportion. Asterisks denote significant difference: ${ }^{* *} p \leq 0.001$. (z-tests, $p$ values were

1228

1229

1230

1231

1232

1233

1234

1235

1236

1237

1238

1239

1240

1241

1242

1243

1244

1245

1246

1247

1248

1249

1250

1251

1252

1253

1254

1255

1256

1257

1258

1259

1260

1261

1262

1263

1264

1265

1266

1267

1268

1269

1270 corrected by multiplying by the number of comparisons, Bonferroni correction).

\section{Fig. 4. SAX-7S is expressed in virtually all neurons throughout life.}

(A) Images of SAX-7::GFP expression reporting both SAX-7L and SAX-7S. As shown on the schematics, in this previously published transgene (Sarov et al., 2012), the gene coding for EGFP was inserted into the gene sax-7 by fosmid recombineering in such a way that both SAX-7S and SAX-7L isoforms were tagged, making impossible to distinguish between them. SAX-7::GFP is broadly expressed in neurons and epidermal cells (vulval cells, seam cells). (B) Confocal images showing sfGFP::SAX-7S expression. As shown on the schematics, the gene coding for sfGFP was inserted by CRISPR-Cas 9 at the end of exon 1 of sax-7S in order to specifically tag SAX-7S (see Fig. S1D; qv31 in Table 1). "sfGFP", superfolderGFP; "SP", export signal peptide sequence part of sax-7S inserted along with sfgfp. (B') Untreated confocal image of a late $4^{\text {th }}$ larval stage worm. Arrows indicate neurons of ventral nerve cord and arrowheads point to examples of background green auto-florescence due to gut granules. Dotted boxes indicate the body region (head or tail) analyzed in B". (B") Images of animals at the indicated larval stages and days of adulthood, examined by confocal microscopy followed by unmixing. Aged worms ( $>5$-days old) have notably increased background autofluorescence. Arrows indicate sfGFP::SAX-7S expression in neurons of the head (left) or tail (right) ganglia. $\mathrm{n} \geq 20$ animals examined by confocal microscopy for each stage. $z$-stack projections. Scale bar, $10 \mu \mathrm{m}$.

Fig. 5. The two SAX-7S cleavage products derived from the serine protease cleavage site, together, can mediate the maintenance of neuronal architecture.

(A) Schematics of full-length and recombinant transgenic versions of SAX-7S used in this study. Blue triangles indicate the signal peptide of SAX-7L. Green triangles indicate the signal peptide of SAX-7S. " $\triangle \lg 3-4$ " contains the entire SAX-7S protein except for the two first Ig domains. " $\triangle \mathrm{lg} 5-6$ " contains the entire SAX-7S protein except for the Ig5 and 6 domains. In " $\triangle$ Fnlll\#3", SAX-7S::Myc lacks the $3^{\text {rd }}$ Fnlll domain. In " $\Delta$ Fnlll", SAX-7S lacks all Fnlll domains. In " $\triangle$ ankyrin", SAX-7S lacks the intracellular ankyrin binding domain. In " $\triangle \mathrm{ICD}$ ", SAX-7S lacks the intracellular domain. "Fragment A" contains the SAX-7S protein region from Ig3 to the serine protease cleavage site (RWKR). "Fragment B" contains the SAX-7S::Myc protein region from the serine protease cleavage site (RWKR) to PDZ::Myc. "Fragment C" contains the SAX-7S protein region from Ig3 to the proximal-transmembrane cleavage site. "Fragment D" contains the SAX-7S protein region from the proximal-transmembrane cleavage site to PDZ.

"Ig", Immunoglobulin-like domain; "FnIII", Fibronectin type III domain; "F", FERM domain binding motif; "A", Ankyrin binding motif; "P", PDZ domain binding motif; bold violet line indicates the transmembrane domain; red arrows indicate serine protease cleavage site in Fnlll\#3 or, cleavage site close to the transmembrane domain.

(B) Western blot analysis of wild-type animals (+), sax-7(qv30) null mutants expressing transgenes for various Myc-tagged SAX-7S fragments. $\mathrm{N}$-terminal and $\mathrm{C}$-terminal fragments of SAX-7S proteins were detected with anti-Myc antibody. Mixed-stage populations of $>5000$ worms were loaded per well, including a variable proportion of animals that actually carry the 
1271 extrachromosomal array (and therefore are transgenic), as the array gets lost randomly upon cell divisions and generations; this comparison is only qualitative. As expected, in lysates of

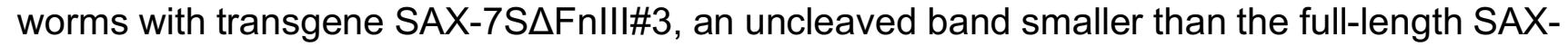
$7 S$ is detected. "C-term products" indicates C-terminal cleavage product, "Ser site" indicates serine protease cleavage site, " TM site" indicates cleavage site near to transmembrane domain. "?" indicates an unknown form of SAX-7S. The three top anti-Myc panels correspond to the same membrane but at different exposure times in order to facilitate the observation of bands that are largely different in abundance (as was done in Fig. 1D). a-HSP90 was used as a loading control.

1280 (C) The defects of qv30 null mutants are rescued by the expression of specific sax-7S(+) variants in the nervous system using the heterologous promoter Punc-14. The relative positioning of the soma and nerve ring axons of chemosensory neurons ASH/ASI (as in Fig. 2A) was evaluated using the reporter Psra-6::DsRed2. Wild-type control and qv30 mutants, along with distinct SAX-7S recombinant transgenic animals, were examined as 1-day adults. Domain analyses are shown on the left of the graph, and fragment analyses on the right, as indicated. The simultaneous absence of $\lg 3$ and 4 fails to rescue, while other domain deletions remain fully or largely functional. For fragment analyses, fragment $A$ and $B$ rescue the defects of the null mutant, indicating that the two SAX-7S protein fragments somehow reconstitute function. Two separate sets of independent extrachromosomal arrays for fragments $C$ and $D$ were tested (C\#1+D\#1, and C\#2+D\#2), which failed to rescue. Sample size is indicated under each column of the graph. Error bars are standard error of the proportion. Asterisks denote significant difference: ${ }^{* * *} p \leq 0.001$. (z-tests, $p$ values were corrected by multiplying by the number of comparisons, Bonferroni correction). "+", indicates wild-type strain; n.s., not significant.

\section{SUPPLEMENTARY FIGURE LEGENDS}

1300

Fig. S1. Sequence information for the four CRISPR-Cas9 genome edited alleles 1302 generated in this study.

1303 (A) Diagram of the qv30 allele. It is a 19973 bp deletion, from base 8364 in exon 1 of sax-7L on cosmid C18F3 (indicated by white dotted line with a red asterisk in exon 1) to base 28337 (indicated by white dotted line with red asterisk in exon 17 of sax-7L, and exon 14 of sax-7S). Below, the exact sequence context of the qv30 deletion is provided (with the red line and asterisks), on what remains of sax-7L exons in blue, and of sax-7S exons in green. Exons of sax-7L and sax-7S are represented by blue and green boxes, respectively, and UTRs by grey boxes.

(B) Diagram of the $q v 25$ allele. It is an insertion of $47 \mathrm{bp}$ (indicated in green), right after bp 12785 exon 1 of sax-7S on C18F3. It creates an ORF frameshift and introduces a stop codon in the sax-7S signal peptide (the signal peptide is encoded by sequence on exon 1 and beginning part of exon 2). sax-7S exons are represented by green boxes and introns by black lines. 
1315 (C) Diagram of the qv26 allele. It is an insertion of $36 \mathrm{bp}$ (indicated in green), right after bp 131612785 exon 1 of sax-7S on C18F3. It disrupts the sax-7S signal peptide (the signal peptide is

1317 encoded by sequence on exon 1 and beginning part of exon 2). sax-7S exons are represented

1318 by green boxes and introns by black lines.

1319 (D) Diagram of the qv31 allele. In order to tag the endogenous short isoform (sax-7S) 1320 specifically, the gene coding for superfolder GFP (sfgfp, $732 \mathrm{bp}$ ), preceded by the 1321 downstream part of the coding sequence for the sax-7S signal peptide (from beginning exon 13222 of sax-7S), was inserted by CRISPR-Cas9-mediated homology repair. This insertion (highlighted in yellow) starts right at the end of exon 1 of sax-7S, after bp 12809 of C18F3. "SP" means sax-7S signal peptide sequence part added. Exons of sax-7L and sax-7S are represented by blue and green boxes, respectively, and introns by black lines.

Fig. S2. Phenotypic characterization of sax-7(qv30).

1329

(A) sax-7 mutants have normal egg laying behavior. Ability to normally lay embryos was examined each day from day 1 to 5 of adulthood.

(B) sax-7 mutants have normal embryonic viability.

(C) sax-7 mutants have a smaller brood size. Quantification of the total number of embryos laid from L4 until 4-day-old in wild type, null mutant qv30, and hypomorphic mutant nj48.

\section{Fig. S3. Other sites of SAX-7S expression.}

(A) Unmixed confocal images showing sfGFP::SAX-7S expression (green fluorescence) in neurons at different embryonic stages. No fluorescence was observed in 28-cell stage embryos (data not shown). White arrows indicate sfGFP::SAX-7S expression in embryonic neurons, localized to the plasma membrane. (B) Unmixed confocal images showing sfGFP::SAX-7S expression (green fluorescence) in the developing reproductive system in late $4^{\text {th }}$ larval stage uterus. sfGFP::SAX-7S expression is seen in the utse syncytium (empty white arrowhead), in two uterine ventral cells (likely uv1, white asterisks) and in neurons of the ventral nerve cord (white arrows) of the worm. $n \geq 20$ animals examined by confocal microscopy for each stage. Z-stack maximum intensity projections. Scale bar, $10 \mu \mathrm{m}$. 
1345

1346

1347

1348

1349

1350

1351

1352

1353

1354

1355

1356

1357

1358

1359

1360

1361

1362

1363

1364

1365

1366

1367

1368

1369

1370

1371

1372

1373

1374

1375

1376

1377

1378

1379

1380

1381

1382

1383

1384

1385

1386

1387

1388

1389

1390

1391

1392

1393

1394

\section{REFERENCES}

Ackermann, C. (2017) (Heidelberg University ).

Appel, F., Holm, J., Conscience, J.F., and Schachner, M. (1993). Several extracellular domains of the neural cell adhesion molecule L1 are involved in neurite outgrowth and cell body adhesion. J Neurosci 13, 4764-4775.

Arribere, J.A., Bell, R.T., Fu, B.X., Artiles, K.L., Hartman, P.S., and Fire, A.Z. (2014). Efficient marker-free recovery of custom genetic modifications with CRISPR/Cas9 in Caenorhabditis elegans. Genetics 198, 837-846.

Aurelio, O., Hall, D.H., and Hobert, O. (2002). Immunoglobulin-domain proteins required for maintenance of ventral nerve cord organization. Science 295, 686-690.

Beer, S., Oleszewski, M., Gutwein, P., Geiger, C., and Altevogt, P. (1999). Metalloproteinasemediated release of the ectodomain of $L 1$ adhesion molecule. J Cell Sci 112 (Pt 16), 26672675.

Benard, C., and Hobert, O. (2009). Looking beyond development: maintaining nervous system architecture. Curr Top Dev Biol 87, 175-194.

Benard, C., Tjoe, N., Boulin, T., Recio, J., and Hobert, O. (2009). The small, secreted immunoglobulin protein ZIG-3 maintains axon position in Caenorhabditis elegans. Genetics 183, 917-927.

Benard, C.Y., Blanchette, C., Recio, J., and Hobert, O. (2012). The secreted immunoglobulin domain proteins ZIG-5 and ZIG-8 cooperate with L1CAM/SAX-7 to maintain nervous system integrity. PLoS Genet 8, e1002819.

Benard, C.Y., Boyanov, A., Hall, D.H., and Hobert, O. (2006). DIG-1, a novel giant protein, nonautonomously mediates maintenance of nervous system architecture. Development 133 , 3329-3340.

Bieber, A.J., Snow, P.M., Hortsch, M., Patel, N.H., Jacobs, J.R., Traquina, Z.R., Schilling, J., and Goodman, C.S. (1989). Drosophila neuroglian: a member of the immunoglobulin superfamily with extensive homology to the vertebrate neural adhesion molecule L1. Cell 59, 447-460.

Blaess, S., Kammerer, R.A., and Hall, H. (1998). Structural analysis of the sixth immunoglobulin-like domain of mouse neural cell adhesion molecule L1 and its interactions with alpha(v)beta3, alpha(Illb)beta3, and alpha5beta1 integrins. J Neurochem 71, 2615-2625.

Brenner, S. (1974). The genetics of Caenorhabditis elegans. Genetics 77, 71-94.

Brummendorf, T., Kenwrick, S., and Rathjen, F.G. (1998). Neural cell recognition molecule L1: from cell biology to human hereditary brain malformations. Curr Opin Neurobiol 8, 87-97.

Brummendorf, T., and Rathjen, F.G. (1996). Structure/function relationships of axon-associated adhesion receptors of the immunoglobulin superfamily. Curr Opin Neurobiol 6, 584-593.

Bülow, H.E., Boulin, T., and Hobert, O. (2004). Differential functions of the C. elegans FGF receptor in axon outgrowth and maintenance of axon position. Neuron 42, 367-374.

Castellani, V., De Angelis, E., Kenwrick, S., and Rougon, G. (2002). Cis and trans interactions of L1 with neuropilin-1 control axonal responses to semaphorin 3A. EMBO J 21, 6348-6357.

Cebul, E.R., McLachlan, I.G., and Heiman, M.G. (2020). Dendrites with specialized glial attachments develop by retrograde extension using SAX-7 and GRDN-1. Development 147.

Chen, C.H., Hsu, H.W., Chang, Y.H., and Pan, C.L. (2019). Adhesive L1CAM-Robo Signaling Aligns Growth Cone F-Actin Dynamics to Promote Axon-Dendrite Fasciculation in C. elegans. Dev Cell 48, 215-228 e215.

Chen, L., Ong, B., and Bennett, V. (2001). LAD-1, the Caenorhabditis elegans L1CAM homologue, participates in embryonic and gonadal morphogenesis and is a substrate for fibroblast growth factor receptor pathway-dependent phosphotyrosine-based signaling. J Cell Biol 154, 841856.

Cherra, S.J., 3rd, and Jin, Y. (2016). A Two-Immunoglobulin-Domain Transmembrane Protein Mediates an Epidermal-Neuronal Interaction to Maintain Synapse Density. Neuron. 
1395

1396

1397

1398

1399

1400

1401

1402

1403

1404

1405

1406

1407

1408

1409

1410

1411

1412

1413

1414

1415

1416

1417

1418

1419

1420

1421

1422

1423

1424

1425

1426

1427

1428

1429

1430

1431

1432

1433

1434

1435

1436

1437

1438

1439

1440

1441

1442

1443

1444

1445

Collet, J., Spike, C.A., Lundquist, E.A., Shaw, J.E., and Herman, R.K. (1998). Analysis of osm-6, a gene that affects sensory cilium structure and sensory neuron function in Caenorhabditis elegans. Genetics 148, 187-200.

Davey, F., Hill, M., Falk, J., Sans, N., and Gunn-Moore, F.J. (2005). Synapse associated protein 102 is a novel binding partner to the cytoplasmic terminus of neurone-glial related cell adhesion molecule. J Neurochem 94, 1243-1253.

Davis, J.Q., and Bennett, V. (1994). Ankyrin binding activity shared by the neurofascin/L1/NrCAM family of nervous system cell adhesion molecules. J Biol Chem 269, 27163-27166.

De Angelis, E., MacFarlane, J., Du, J.S., Yeo, G., Hicks, R., Rathjen, F.G., Kenwrick, S., and Brummendorf, T. (1999). Pathological missense mutations of neural cell adhesion molecule L1 affect homophilic and heterophilic binding activities. EMBO J 18, 4744-4753.

De Angelis, E., Watkins, A., Schafer, M., Brummendorf, T., and Kenwrick, S. (2002). Diseaseassociated mutations in L1 CAM interfere with ligand interactions and cell-surface expression. Hum Mol Genet 11, 1-12.

Desai, C., and Horvitz, H.R. (1989). Caenorhabditis elegans mutants defective in the functioning of the motor neurons responsible for egg laying. Genetics 121, 703-721.

Diaz-Balzac, C.A., Lazaro-Pena, M.I., Ramos-Ortiz, G.A., and Bulow, H.E. (2015). The Adhesion Molecule KAL-1/anosmin-1 Regulates Neurite Branching through a SAX-7/L1CAM-EGL15/FGFR Receptor Complex. Cell Rep 11, 1377-1384.

Diaz-Balzac, C.A., Rahman, M., Lazaro-Pena, M.I., Martin Hernandez, L.A., Salzberg, Y., AguirreChen, C., Kaprielian, Z., and Bulow, H.E. (2016). Muscle- and Skin-Derived Cues Jointly Orchestrate Patterning of Somatosensory Dendrites. Curr Biol 26, 2379-2387.

Dickinson, D.J., Ward, J.D., Reiner, D.J., and Goldstein, B. (2013). Engineering the Caenorhabditis elegans genome using Cas9-triggered homologous recombination. Nat Methods 10, 10281034.

Dirks, P., Thomas, U., and Montag, D. (2006). The cytoplasmic domain of NrCAM binds to PDZ domains of synapse-associated proteins SAP90/PSD95 and SAP97. Eur J Neurosci 24, 2531.

Dong, X., Liu, O.W., Howell, A.S., and Shen, K. (2013). An extracellular adhesion molecule complex patterns dendritic branching and morphogenesis. Cell 155, 296-307.

Faissner, A., Teplow, D.B., Kubler, D., Keilhauer, G., Kinzel, V., and Schachner, M. (1985). Biosynthesis and membrane topography of the neural cell adhesion molecule L1. EMBO J 4, 3105-3113.

Falk, J., Thoumine, O., Dequidt, C., Choquet, D., and Faivre-Sarrailh, C. (2004). NrCAM coupling to the cytoskeleton depends on multiple protein domains and partitioning into lipid rafts. Mol Biol Cell 15, 4695-4709.

Felding-Habermann, B., Silletti, S., Mei, F., Siu, C.H., Yip, P.M., Brooks, P.C., Cheresh, D.A., O'Toole, T.E., Ginsberg, M.H., and Montgomery, A.M. (1997). A single immunoglobulin-like domain of the human neural cell adhesion molecule $L 1$ supports adhesion by multiple vascular and platelet integrins. J Cell Biol 139, 1567-1581.

Fire, A., Harrison, S.W., and Dixon, D. (1990). A modular set of lacZ fusion vectors for studying gene expression in Caenorhabditis elegans. Gene 93, 189-198.

Fransen, E., Van Camp, G., Vits, L., and Willems, P.J. (1997). L1-associated diseases: clinical geneticists divide, molecular geneticists unite. Hum Mol Genet 6, 1625-1632.

Gil, O.D., Sakurai, T., Bradley, A.E., Fink, M.Y., Cassella, M.R., Kuo, J.A., and Felsenfeld, D.P. (2003). Ankyrin binding mediates L1CAM interactions with static components of the cytoskeleton and inhibits retrograde movement of L1CAM on the cell surface. J Cell Biol 162, 719-730.

Godenschwege, T.A., Kristiansen, L.V., Uthaman, S.B., Hortsch, M., and Murphey, R.K. (2006). A conserved role for Drosophila Neuroglian and human L1-CAM in central-synapse formation. Curr Biol 16, 12-23. 
1446

1447

1448

1449

1450

1451

1452

1453

1454

1455

1456

1457

1458

1459

1460

1461

1462

1463

1464

1465

1466

1467

1468

1469

1470

1471

1472

1473

1474

1475

1476

1477

1478

1479

1480

1481

1482

1483

1484

1485

1486

1487

1488

1489

1490

1491

1492

1493

1494

1495

1496

Gunn-Moore, F.J., Hill, M., Davey, F., Herron, L.R., Tait, S., Sherman, D., and Brophy, P.J. (2006). A functional FERM domain binding motif in neurofascin. Mol Cell Neurosci 33, 441-446.

Gutwein, P., Mechtersheimer, S., Riedle, S., Stoeck, A., Gast, D., Joumaa, S., Zentgraf, H., Fogel, M., and Altevogt, D.P. (2003). ADAM10-mediated cleavage of L1 adhesion molecule at the cell surface and in released membrane vesicles. FASEB J 17, 292-294.

Hall, S.G., and Bieber, A.J. (1997). Mutations in the Drosophila neuroglian cell adhesion molecule affect motor neuron pathfinding and peripheral nervous system patterning. J Neurobiol 32, 325-340.

Haspel, J., Friedlander, D.R., Ivgy-May, N., Chickramane, S., Roonprapunt, C., Chen, S., Schachner, M., and Grumet, M. (2000). Critical and optimal Ig domains for promotion of neurite outgrowth by L1/Ng-CAM. J Neurobiol 42, 287-302.

Haspel, J., and Grumet, M. (2003). The L1CAM extracellular region: a multi-domain protein with modular and cooperative binding modes. Front Biosci 8, s1210-1225.

Hedgecock, E.M., Culotti, J.G., Thomson, J.N., and Perkins, L.A. (1985). Axonal guidance mutants of Caenorhabditis elegans identified by filling sensory neurons with fluorescein dyes. Dev Biol 111, 158-170.

Heiman, M.G., and Pallanck, L. (2011). Neurons at the extremes of cell biology. Mol Biol Cell 22, 721.

Herron, L.R., Hill, M., Davey, F., and Gunn-Moore, F.J. (2009). The intracellular interactions of the L1 family of cell adhesion molecules. Biochem J 419, 519-531.

Holm, J., Appel, F., and Schachner, M. (1995). Several extracellular domains of the neural cell adhesion molecule L1 are involved in homophilic interactions. J Neurosci Res 42, 9-20.

Hoogewijs, D., Houthoofd, K., Matthijssens, F., Vandesompele, J., and Vanfleteren, J.R. (2008). Selection and validation of a set of reliable reference genes for quantitative sod gene expression analysis in C. elegans. BMC molecular biology 9, 9.

Hortsch, M. (1996). The L1 family of neural cell adhesion molecules: old proteins performing new tricks. Neuron 17, 587-593.

Hortsch, M. (2000). Structural and functional evolution of the L1 family: are four adhesion molecules better than one? Mol Cell Neurosci 15, 1-10.

Hortsch, M., Nagaraj, K., and Mualla, R. (2014). The L1 family of cell adhesion molecules: a sickening number of mutations and protein functions. Adv Neurobiol 8, 195-229.

Jafari, G., Burghoorn, J., Kawano, T., Mathew, M., Morck, C., Axang, C., Ailion, M., Thomas, J.H., Culotti, J.G., Swoboda, P., et al. (2010). Genetics of extracellular matrix remodeling during organ growth using the Caenorhabditis elegans pharynx model. Genetics 186, 969-982.

Johnson, R.P., and Kramer, J.M. (2012). Neural maintenance roles for the matrix receptor dystroglycan and the nuclear anchorage complex in Caenorhabditis elegans. Genetics 190, 1365-1377.

Jones, D., Russnak, R.H., Kay, R.J., and Candido, E.P. (1986). Structure, expression, and evolution of a heat shock gene locus in Caenorhabditis elegans that is flanked by repetitive elements. J Biol Chem 261, 12006-12015.

Kalus, I., Schnegelsberg, B., Seidah, N.G., Kleene, R., and Schachner, M. (2003). The proprotein convertase PC5A and a metalloprotease are involved in the proteolytic processing of the neural adhesion molecule L1. J Biol Chem 278, $10381-10388$.

Kiefel, H., Bondong, S., Hazin, J., Ridinger, J., Schirmer, U., Riedle, S., and Altevogt, P. (2012). L1CAM: a major driver for tumor cell invasion and motility. Cell adhesion \& migration 6, 374384.

Kleene, R., Lutz, D., Loers, G., Bork, U., Borgmeyer, U., Hermans-Borgmeyer, I., and Schachner, M. (2020). Revisiting the proteolytic processing of cell adhesion molecule L1. J Neurochem.

Kolata, S., Wu, J., Light, K., Schachner, M., and Matzel, L.D. (2008). Impaired working memory duration but normal learning abilities found in mice that are conditionally deficient in the close homolog of L1. J Neurosci 28, 13505-13510. 
1497

1498

1499

1500

1501

1502

1503

1504

1505

1506

1507

1508

1509

1510

1511

1512

1513

1514

1515

1516

1517

1518

1519

1520

1521

1522

1523

1524

1525

1526

1527

1528

1529

1530

1531

1532

1533

1534

1535

1536

1537

1538

1539

1540

1541

1542

1543

1544

1545

1546

1547

Koroll, M., Rathjen, F.G., and Volkmer, H. (2001). The neural cell recognition molecule neurofascin interacts with syntenin-1 but not with syntenin-2, both of which reveal self-associating activity. J Biol Chem 276, 10646-10654.

Koticha, D., Babiarz, J., Kane-Goldsmith, N., Jacob, J., Raju, K., and Grumet, M. (2005). Cell adhesion and neurite outgrowth are promoted by neurofascin NF155 and inhibited by NF186. Mol Cell Neurosci 30, 137-148.

Kriebel, M., Metzger, J., Trinks, S., Chugh, D., Harvey, R.J., Harvey, K., and Volkmer, H. (2011). The cell adhesion molecule neurofascin stabilizes axo-axonic GABAergic terminals at the axon initial segment. J Biol Chem 286, 24385-24393.

Kunz, S., Spirig, M., Ginsburg, C., Buchstaller, A., Berger, P., Lanz, R., Rader, C., Vogt, L., Kunz, B., and Sonderegger, P. (1998). Neurite fasciculation mediated by complexes of axonin-1 and $\mathrm{Ng}$ cell adhesion molecule. J Cell Biol 143, 1673-1690.

Law, J.W., Lee, A.Y., Sun, M., Nikonenko, A.G., Chung, S.K., Dityatev, A., Schachner, M., and Morellini, F. (2003). Decreased anxiety, altered place learning, and increased CA1 basal excitatory synaptic transmission in mice with conditional ablation of the neural cell adhesion molecule L1. J Neurosci 23, 10419-10432.

Linneberg, C., Toft, C.L.F., Kjaer-Sorensen, K., and Laursen, L.S. (2019). L1cam-mediated developmental processes of the nervous system are differentially regulated by proteolytic processing. Scientific reports 9, 3716 .

Lutz, D., Sharaf, A., Drexler, D., Kataria, H., Wolters-Eisfeld, G., Brunne, B., Kleene, R., Loers, G., Frotscher, M., and Schachner, M. (2017). Proteolytic cleavage of transmembrane cell adhesion molecule $L 1$ by extracellular matrix molecule Reelin is important for mouse brain development. Scientific reports 7, 15268.

Lutz, D., Wolters-Eisfeld, G., Joshi, G., Djogo, N., Jakovcevski, I., Schachner, M., and Kleene, R. (2012). Generation and nuclear translocation of sumoylated transmembrane fragment of cell adhesion molecule L1. J Biol Chem 287, 17161-17175.

Maretzky, T., Schulte, M., Ludwig, A., Rose-John, S., Blobel, C., Hartmann, D., Altevogt, P., Saftig, P., and Reiss, K. (2005). L1 is sequentially processed by two differently activated metalloproteases and presenilin/gamma-secretase and regulates neural cell adhesion, cell migration, and neurite outgrowth. Mol Cell Biol 25, 9040-9053.

Maten, M.V., Reijnen, C., Pijnenborg, J.M.A., and Zegers, M.M. (2019). L1 Cell Adhesion Molecule in Cancer, a Systematic Review on Domain-Specific Functions. International journal of molecular sciences 20.

Matsumoto-Miyai, K., Ninomiya, A., Yamasaki, H., Tamura, H., Nakamura, Y., and Shiosaka, S. (2003). NMDA-dependent proteolysis of presynaptic adhesion molecule $L 1$ in the hippocampus by neuropsin. J Neurosci 23, 7727-7736.

Mechtersheimer, S., Gutwein, P., Agmon-Levin, N., Stoeck, A., Oleszewski, M., Riedle, S., Postina, R., Fahrenholz, F., Fogel, M., Lemmon, V., et al. (2001). Ectodomain shedding of L1 adhesion molecule promotes cell migration by autocrine binding to integrins. J Cell Biol 155, 661-673.

Mello, C., and Fire, A. (1995). DNA transformation. Methods Cell Biol 48, 451-482.

Montgomery, A.M., Becker, J.C., Siu, C.H., Lemmon, V.P., Cheresh, D.A., Pancook, J.D., Zhao, X., and Reisfeld, R.A. (1996). Human neural cell adhesion molecule L1 and rat homologue NILE are ligands for integrin alpha $v$ beta 3 . J Cell Biol 132, 475-485.

Naus, S., Richter, M., Wildeboer, D., Moss, M., Schachner, M., and Bartsch, J.W. (2004). Ectodomain shedding of the neural recognition molecule CHL1 by the metalloproteasedisintegrin ADAM8 promotes neurite outgrowth and suppresses neuronal cell death. J Biol Chem 279, 16083-16090.

Nayeem, N., Silletti, S., Yang, X., Lemmon, V.P., Reisfeld, R.A., Stallcup, W.B., and Montgomery, A.M. (1999). A potential role for the plasmin(ogen) system in the posttranslational cleavage of the neural cell adhesion molecule L1. J Cell Sci 112 (Pt 24), 4739-4749. 
1548

1549

1550

1551

1552

1553

1554

1555

1556

1557

1558

1559

1560

1561

1562

1563

1564

1565

1566

1567

1568

1569

1570

1571

1572

1573

1574

1575

1576

1577

1578

1579

1580

1581

1582

1583

1584

1585

1586

1587

1588

1589

1590

1591

1592

1593

1594

1595

1596

1597

1598

Nonet, M.L., Staunton, J.E., Kilgard, M.P., Fergestad, T., Hartwieg, E., Horvitz, H.R., Jorgensen, E.M., and Meyer, B.J. (1997). Caenorhabditis elegans rab-3 mutant synapses exhibit impaired function and are partially depleted of vesicles. J Neurosci 17, 8061-8073.

Ogura, K., Shirakawa, M., Barnes, T.M., Hekimi, S., and Ohshima, Y. (1997). The UNC-14 protein required for axonal elongation and guidance in Caenorhabditis elegans interacts with the serine/threonine kinase UNC-51. Genes Dev 11, 1801-1811.

Oleszewski, M., Beer, S., Katich, S., Geiger, C., Zeller, Y., Rauch, U., and Altevogt, P. (1999). Integrin and neurocan binding to L1 involves distinct Ig domains. J Biol Chem 274, 2460224610.

Pedelacq, J.D., Cabantous, S., Tran, T., Terwilliger, T.C., and Waldo, G.S. (2006). Engineering and characterization of a superfolder green fluorescent protein. Nat Biotechnol 24, 79-88.

Pocock, R., Benard, C.Y., Shapiro, L., and Hobert, O. (2008). Functional dissection of the C. elegans cell adhesion molecule SAX-7, a homologue of human L1. Mol Cell Neurosci 37, 5668.

Rahe, D., Carrera, I., Cosmanescu, F., and Hobert, O. (2019). An isoform-specific allele of the sax-7 locus. microPublication Biology.

Ramirez-Suarez, N.J., Belalcazar, H.M., Salazar, C.J., Beyaz, B., Raja, B., Nguyen, K.C.Q., Celestrin, K., Fredens, J., Faergeman, N.J., Hall, D.H., et al. (2019). Axon-Dependent Patterning and Maintenance of Somatosensory Dendritic Arbors. Dev Cell 48, 229-244 e224.

Riedle, S., Kiefel, H., Gast, D., Bondong, S., Wolterink, S., Gutwein, P., and Altevogt, P. (2009). Nuclear translocation and signalling of L1-CAM in human carcinoma cells requires ADAM10 and presenilin/gamma-secretase activity. Biochem J 420, 391-402.

Rougon, G., and Hobert, O. (2003). New insights into the diversity and function of neuronal immunoglobulin superfamily molecules. Annu Rev Neurosci 26, 207-238.

Sadoul, K., Sadoul, R., Faissner, A., and Schachner, M. (1988). Biochemical characterization of different molecular forms of the neural cell adhesion molecule L1. J Neurochem 50, 510-521.

Salzberg, Y., Diaz-Balzac, C.A., Ramirez-Suarez, N.J., Attreed, M., Tecle, E., Desbois, M., Kaprielian, Z., and Bulow, H.E. (2013). Skin-derived cues control arborization of sensory dendrites in Caenorhabditis elegans. Cell 155, 308-320.

Sarafi-Reinach, T.R., Melkman, T., Hobert, O., and Sengupta, P. (2001). The lin-11 LIM homeobox gene specifies olfactory and chemosensory neuron fates in C. elegans. Development 128, 3269-3281.

Sarov, M., Murray, J.I., Schanze, K., Pozniakovski, A., Niu, W., Angermann, K., Hasse, S., Rupprecht, M., Vinis, E., Tinney, M., et al. (2012). A genome-scale resource for in vivo tagbased protein function exploration in C. elegans. Cell 150, 855-866.

Sasakura, H., Inada, H., Kuhara, A., Fusaoka, E., Takemoto, D., Takeuchi, K., and Mori, I. (2005). Maintenance of neuronal positions in organized ganglia by SAX-7, a Caenorhabditis elegans homologue of L1. Embo J 24, 1477-1488.

Schaefer, A.W., Kamei, Y., Kamiguchi, H., Wong, E.V., Rapoport, I., Kirchhausen, T., Beach, C.M., Landreth, G., Lemmon, S.K., and Lemmon, V. (2002). L1 endocytosis is controlled by a phosphorylation-dephosphorylation cycle stimulated by outside-in signaling by L1. J Cell Biol 157, 1223-1232.

Schafer, M.K., and Altevogt, P. (2010). L1CAM malfunction in the nervous system and human carcinomas. Cell Mol Life Sci 67, 2425-2437.

Schafer, M.K., and Frotscher, M. (2012). Role of L1CAM for axon sprouting and branching. Cell Tissue Res 349, 39-48.

Schmitz, C., Wacker, I., and Hutter, H. (2008). The Fat-like cadherin CDH-4 controls axon fasciculation, cell migration and hypodermis and pharynx development in Caenorhabditis elegans. Dev Biol 316, 249-259.

Sherry, T., Handley, A., Nicholas, H.R., and Pocock, R. (2020). Harmonization of L1CAM expression facilitates axon outgrowth and guidance of a motor neuron. Development 147. 
1599

1600

1601

1602

1603

1604

1605

1606

1607

1608

1609

1610

1611

1612

1613

1614

1615

1616

1617

1618

1619

1620

1621

1622

1623

1624

1625

1626

1627

1628

1629

1630

1631

1632

1633

1634

1635

1636

1637

1638

1639

1640

1641

1642

1643

1644

1645

1646

1647

1648

Silletti, S., Mei, F., Sheppard, D., and Montgomery, A.M. (2000). Plasmin-sensitive dibasic sequences in the third fibronectin-like domain of L1-cell adhesion molecule (CAM) facilitate homomultimerization and concomitant integrin recruitment. J Cell Biol 149, 1485-1502.

Sonderegger, P., Kunz, S., Rader, C., Buchstaller, A., Berger, P., Vogt, L., Kozlov, S.V., Ziegler, U., Kunz, B., Fitzli, D., et al. (1998). Discrete clusters of axonin-1 and NgCAM at neuronal contact sites: facts and speculations on the regulation of axonal fasciculation. Progress in brain research 117, 93-104.

Stringham, E.G., Dixon, D.K., Jones, D., and Candido, E.P. (1992). Temporal and spatial expression patterns of the small heat shock (hsp16) genes in transgenic Caenorhabditis elegans. Mol Biol Cell 3, 221-233.

Tatti, O., Gucciardo, E., Pekkonen, P., Holopainen, T., Louhimo, R., Repo, P., Maliniemi, P., Lohi, J., Rantanen, V., Hautaniemi, S., et al. (2015). MMP16 Mediates a Proteolytic Switch to Promote Cell-Cell Adhesion, Collagen Alignment, and Lymphatic Invasion in Melanoma. Cancer research 75, 2083-2094.

Trent, C., Tsuing, N., and Horvitz, H.R. (1983). Egg-laying defective mutants of the nematode Caenorhabditis elegans. Genetics 104, 619-647.

Wang, X., Kweon, J., Larson, S., and Chen, L. (2005). A role for the C. elegans L1CAM homologue lad-1/sax-7 in maintaining tissue attachment. Dev Biol 284, 273-291.

White, J.G., Southgate, E., Thomson, J.N., and Brenner, S. (1986a). The structure of the nervous system of the nematode Caenorhabditis elegans. Philosophical Transactions of the Royal Society of London B Biological Sciences 314, 1-340.

White, J.G., Southgate, E., Thomson, J.N., and Brenner, S. (1986b). The structure of the nervous system of the nematode Caenorhabditis elegans. Philosophical transactions of the Royal Society of London Series B, Biological sciences 314, 1-340.

Wong, E.V., Cheng, G., Payne, H.R., and Lemmon, V. (1995a). The cytoplasmic domain of the cell adhesion molecule L1 is not required for homophilic adhesion. Neurosci Lett 200, 155-158.

Wong, E.V., Kenwrick, S., Willems, P., and Lemmon, V. (1995b). Mutations in the cell adhesion molecule L1 cause mental retardation. Trends in neurosciences 18, 168-172.

Xu, Y.Z., Ji, Y., Zipser, B., Jellies, J., Johansen, K.M., and Johansen, J. (2003). Proteolytic cleavage of the ectodomain of the L1 CAM family member Tractin. J Biol Chem 278, 4322-4330.

Yip, Z.C., and Heiman, M.G. (2018). Ordered arrangement of dendrites within a C. elegans sensory nerve bundle. eLife 7.

Zallen, J.A., Kirch, S.A., and Bargmann, C.I. (1999). Genes required for axon pathfinding and extension in the C. elegans nerve ring. Development 126, 3679-3692.

Zhao, X., and Siu, C.H. (1995). Colocalization of the homophilic binding site and the neuritogenic activity of the cell adhesion molecule L1 to its second Ig-like domain. J Biol Chem 270, 29413-29421.

Zhao, X., Yip, P.M., and Siu, C.H. (1998). Identification of a homophilic binding site in immunoglobulin-like domain 2 of the cell adhesion molecule L1. J Neurochem 71, 960-971.

Zhou, L., Barao, S., Laga, M., Bockstael, K., Borgers, M., Gijsen, H., Annaert, W., Moechars, D., Mercken, M., Gevaert, K., et al. (2012). The neural cell adhesion molecules L1 and CHL1 are cleaved by BACE1 protease in vivo. J Biol Chem 287, 25927-25940.

Zhou, S., Opperman, K., Wang, X., and Chen, L. (2008). unc-44 Ankyrin and stn-2 gammasyntrophin regulate sax-7 L1CAM function in maintaining neuronal positioning in Caenorhabditis elegans. Genetics 180, 1429-1443.

Zhu, T., Liang, X., Wang, X.M., and Shen, K. (2017). Dynein and EFF-1 control dendrite morphology by regulating the localization pattern of SAX-7 in epidermal cells. J Cell Sci 130, 4063-4071.

Zonta, B., Desmazieres, A., Rinaldi, A., Tait, S., Sherman, D.L., Nolan, M.F., and Brophy, P.J. (2011). A critical role for Neurofascin in regulating action potential initiation through maintenance of the axon initial segment. Neuron 69, 945-956. 


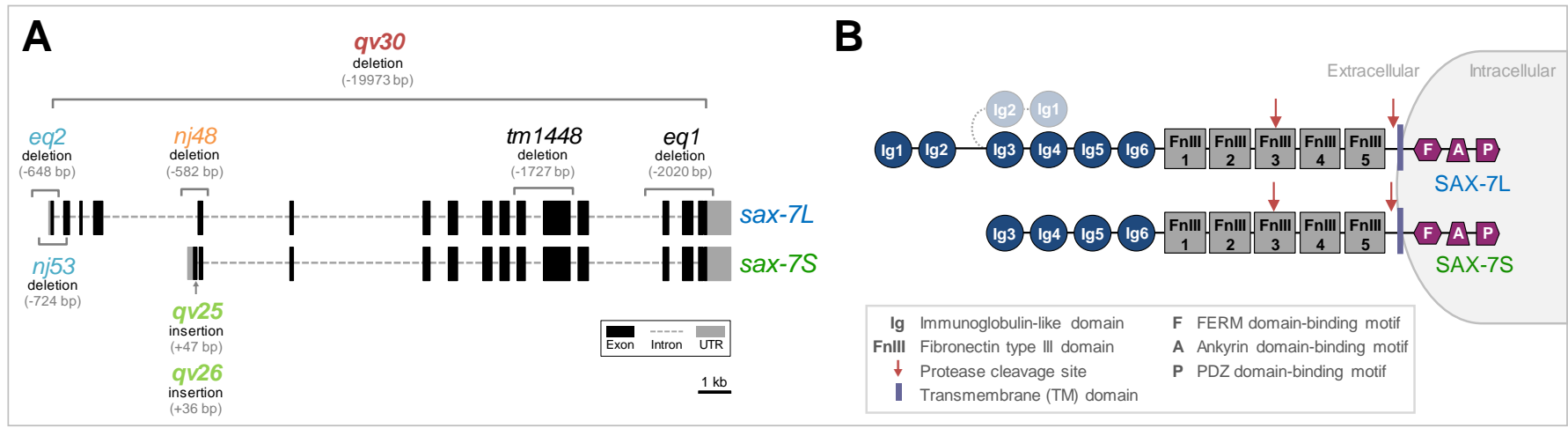

C

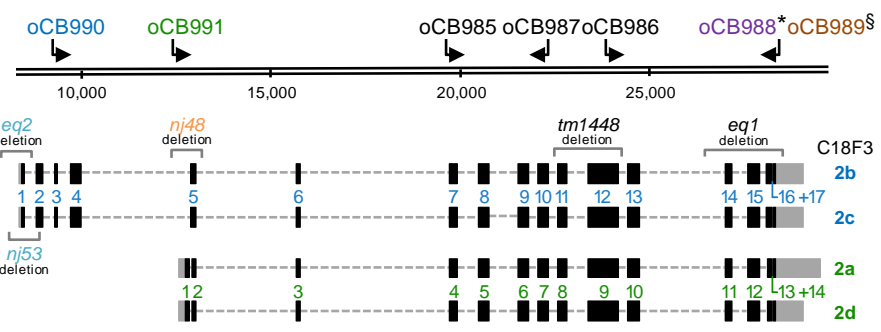

$1 \mathrm{~kb}$

D

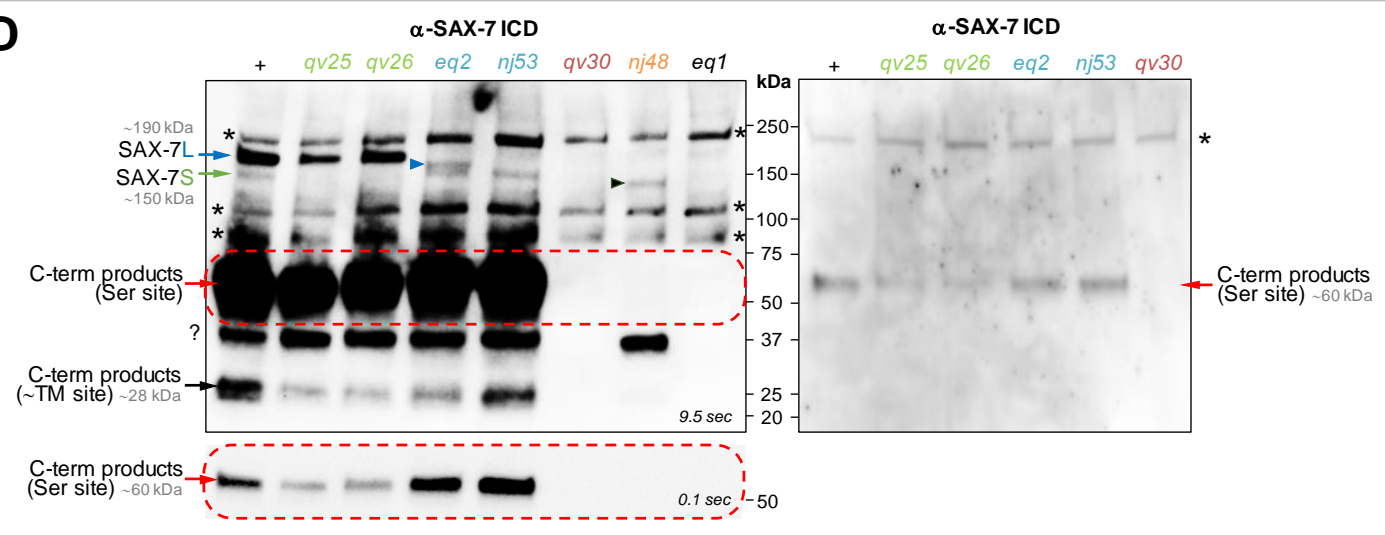

$\alpha$-HSP90

$\alpha$-HSP90

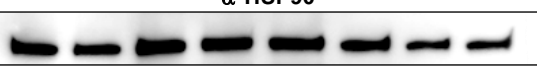

Mixed-stage population of worms

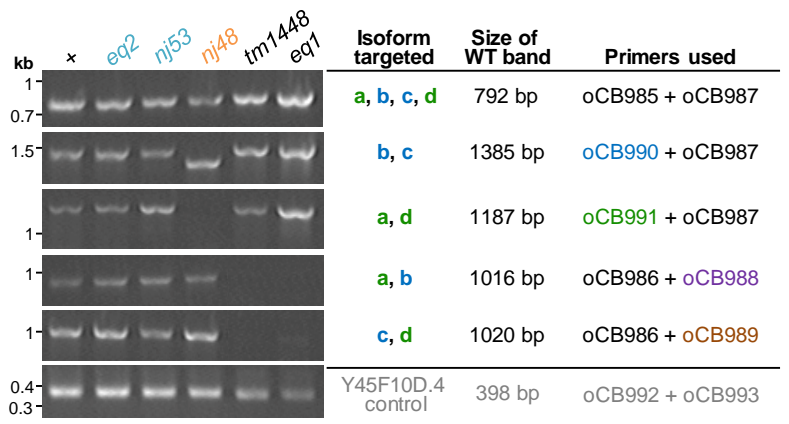

$\alpha$-SAX-7 ICD

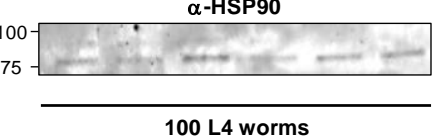

Figure 1 

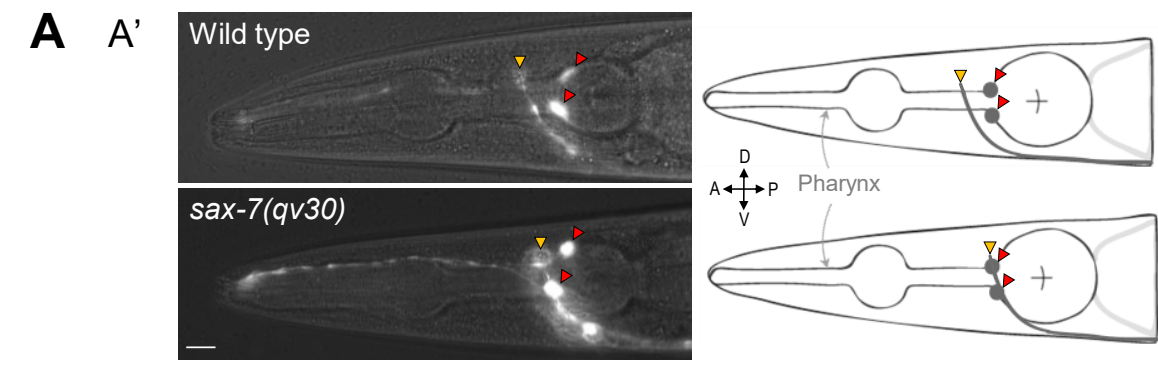

$\underset{\mathrm{V}}{\stackrel{\mathrm{A}}{\longrightarrow} \mathrm{P}}$ Pharynx
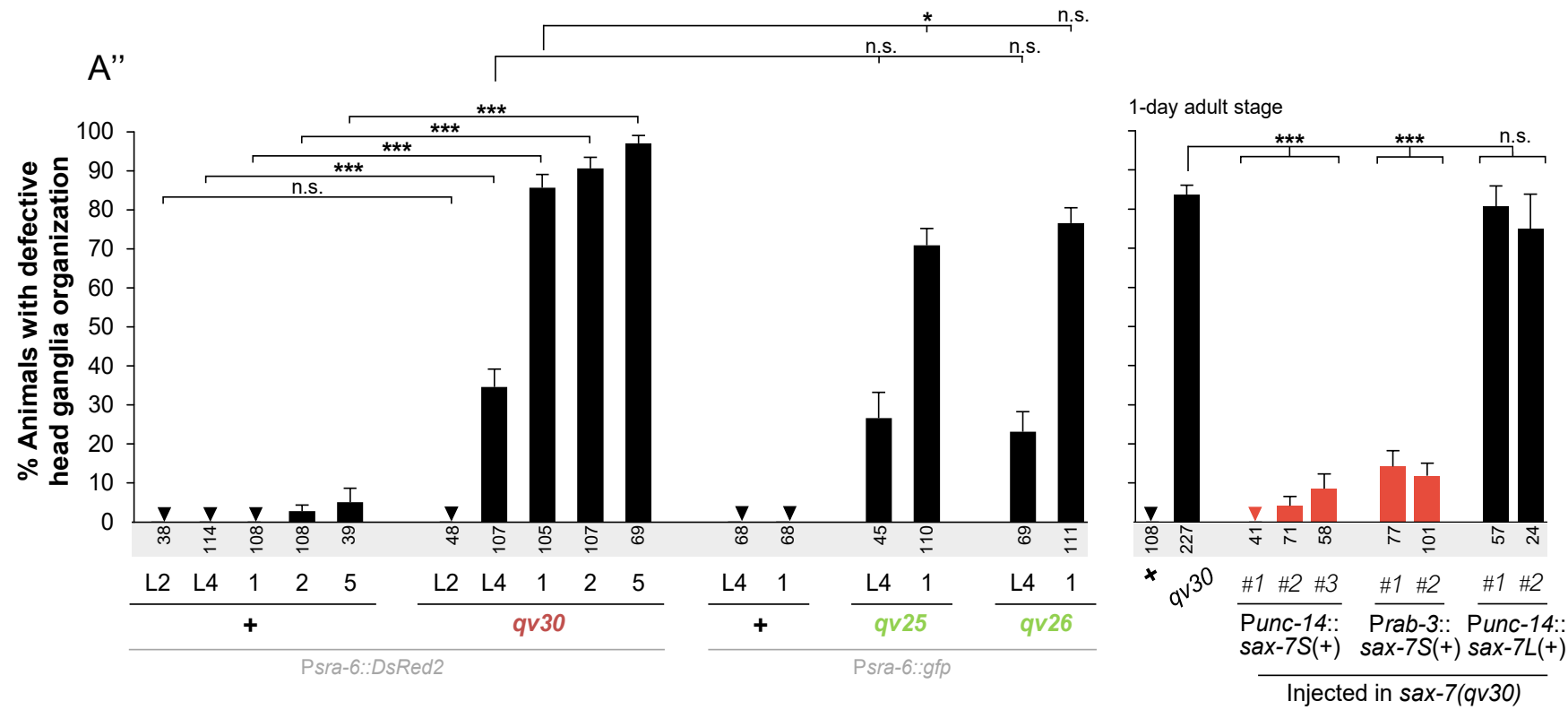

B

B'
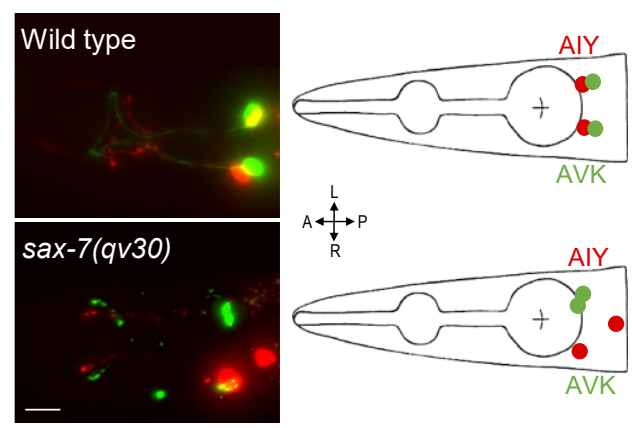

$A \stackrel{L}{\stackrel{L}{\leftrightarrow} P}$
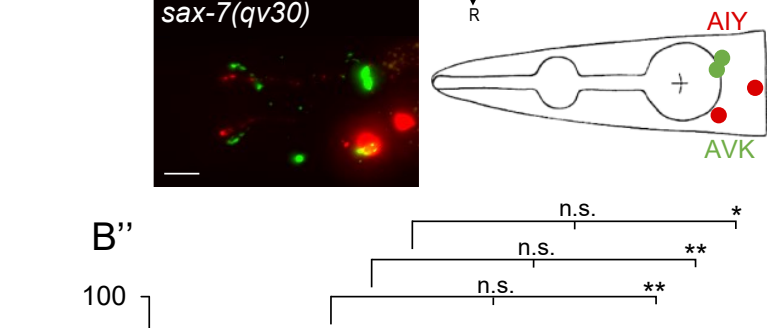

.

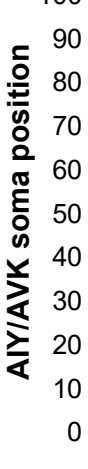

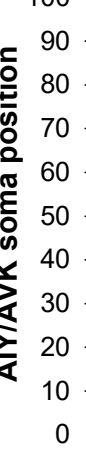

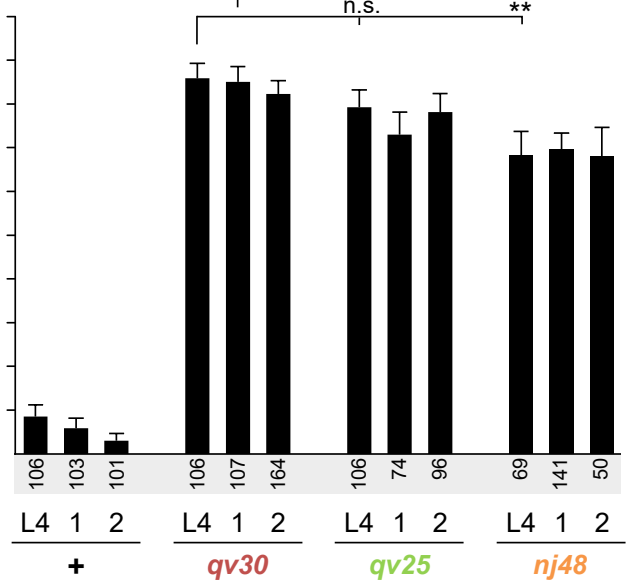

C C' Wild type

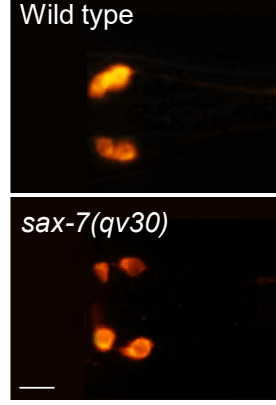

PHA PHB

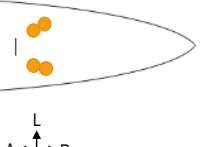

C"

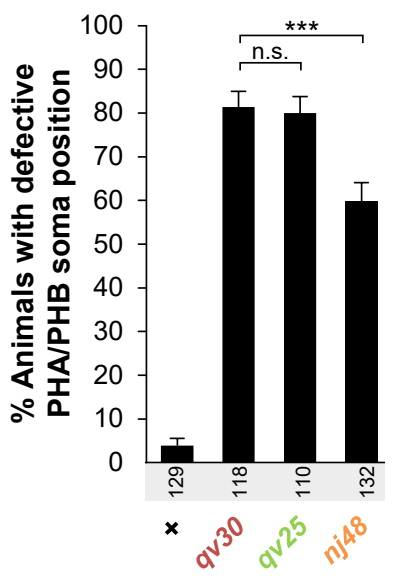

Figure 2 
A Insertion into fosmid

(long and short isoforms tagged)

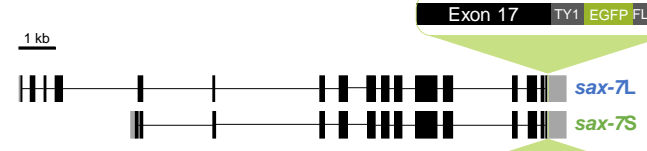

EXon 14 TY1 EGFP FLAG

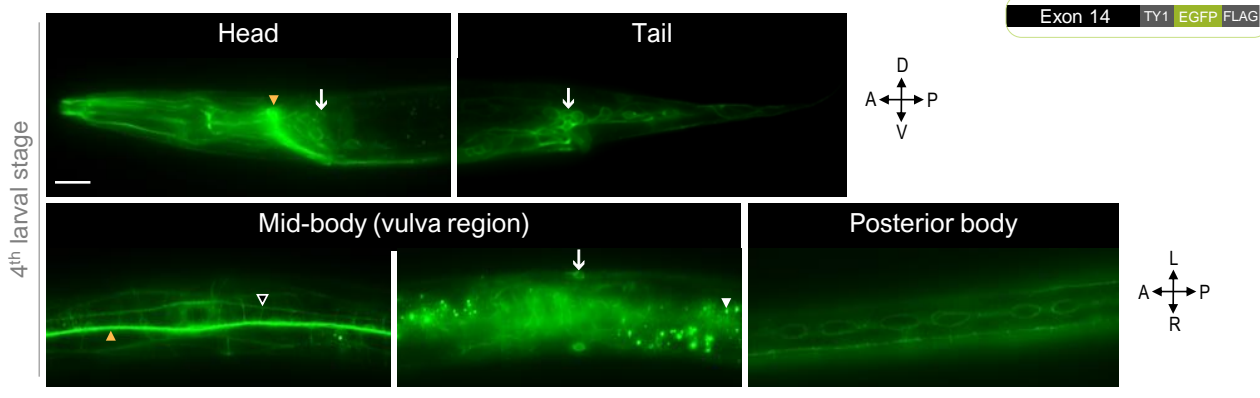

B Insertion at sax-7S endogenous locus (short isoform tagged)

HH | | H HH | H| $\operatorname{sax}-\pi$

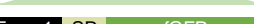

Exon 1 SP SfGFP

$B^{\prime}$

Untreated image

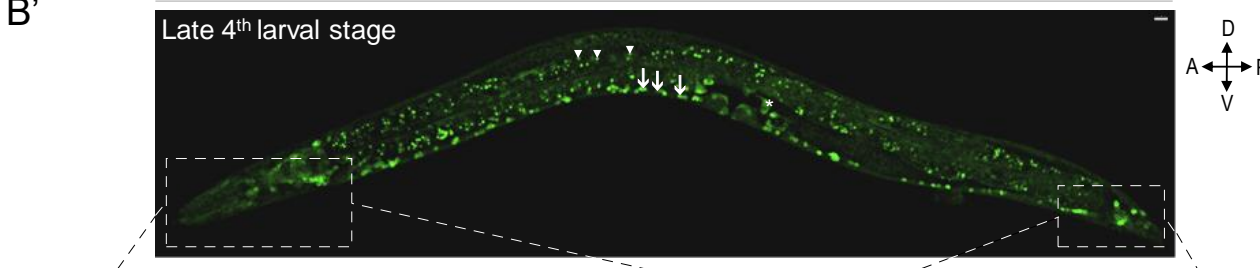

B"

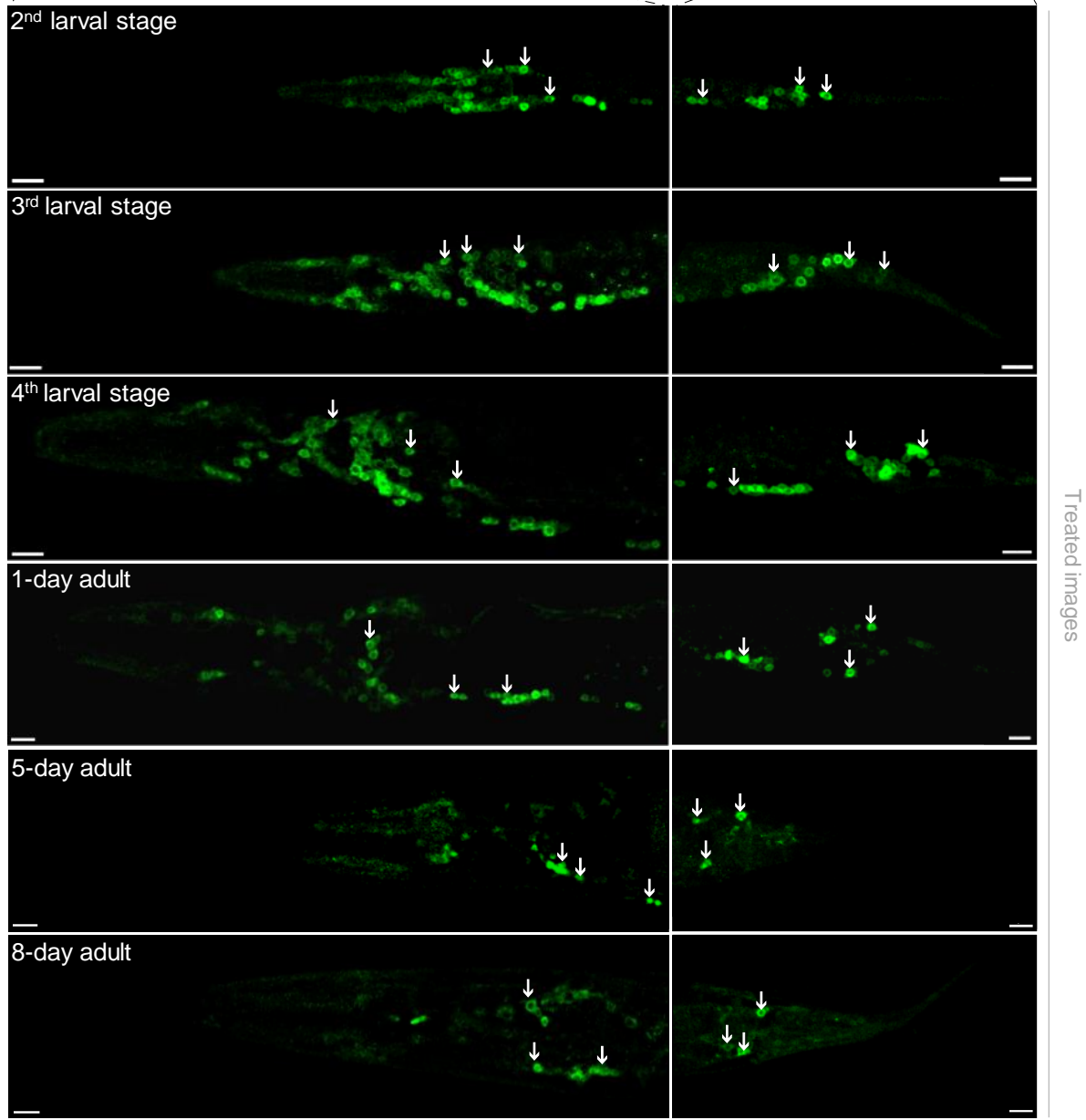

Figure 4 Desse-Bénard 


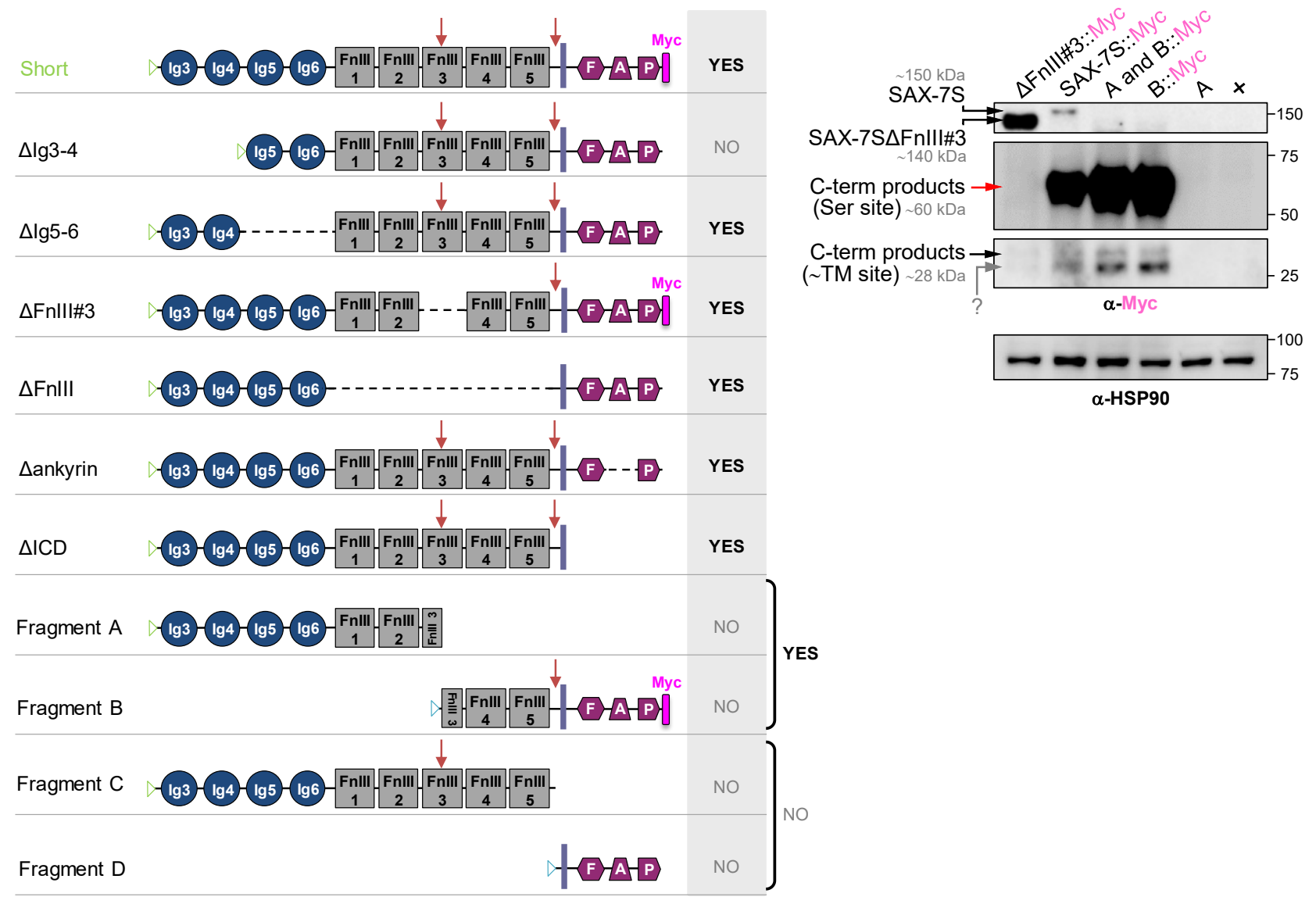

C 1-day adult stage

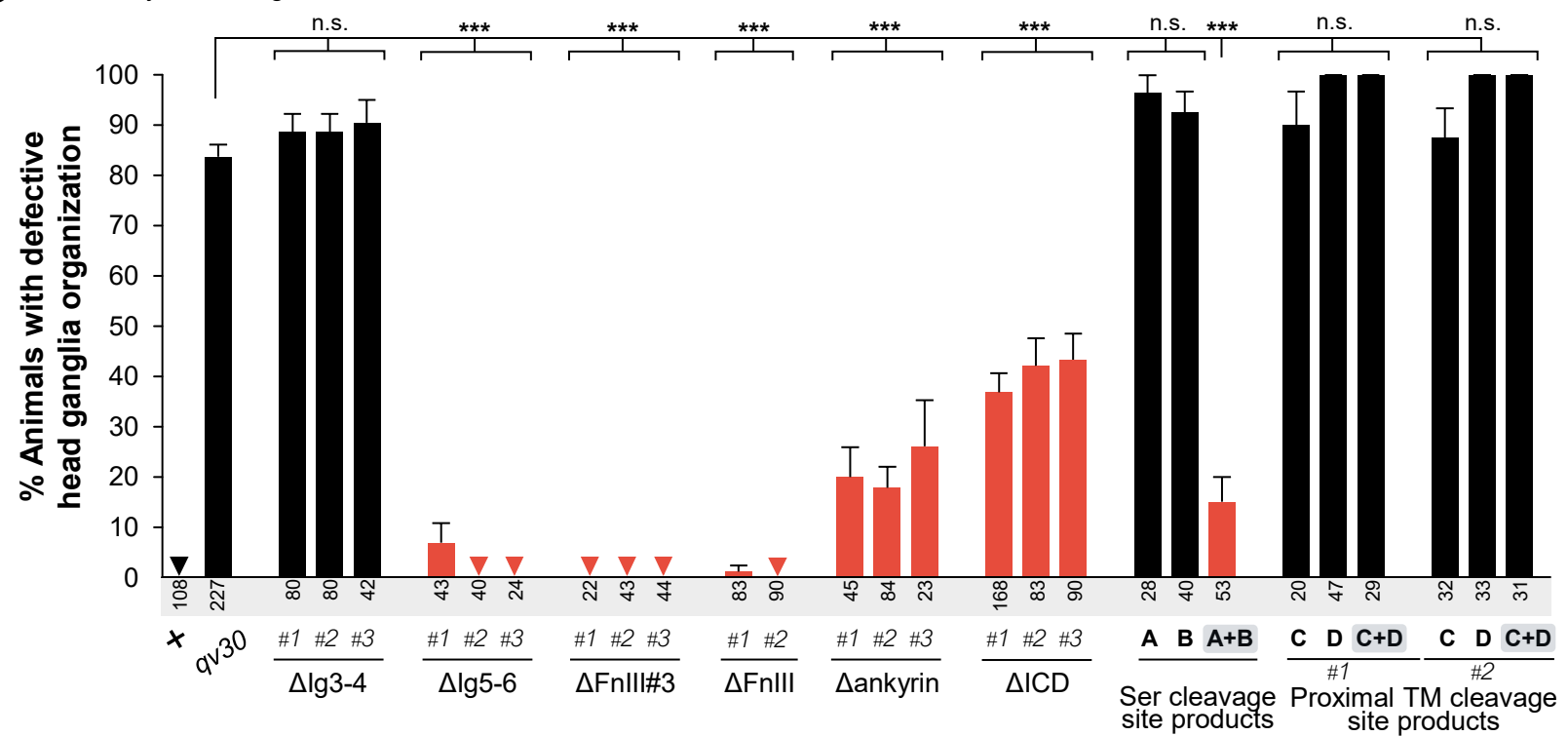


A $q v 30$

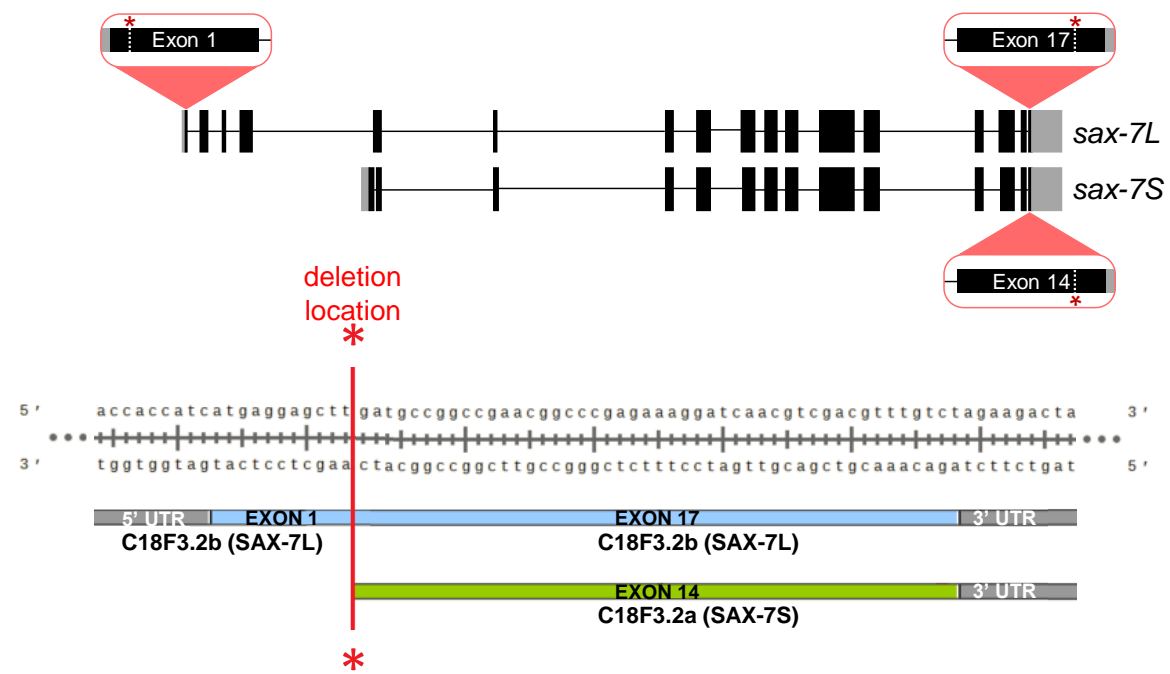

B qv25

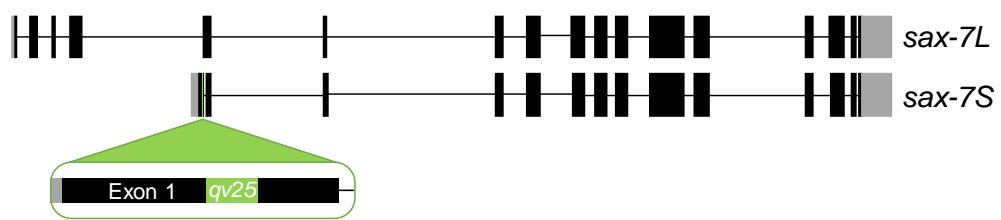

qv25 insertion sequence

STOP

5.

ttcctgatgagCAAAGgaGAAGAACTAGGACGTGCATGGATGAGCTCTACAAACtaggatgtctactgetectegtggtt 3.

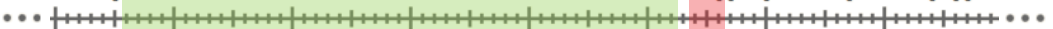

aaggacTACTCGTTTCCTCTTCTTGATCCTGCACGTACCTACTCGAGATGTTTgatCCtacagatgacaggaacaccaa

C18F3.2b (SAX-7L)

C18F3.2a (SAX-7S)

C qv26

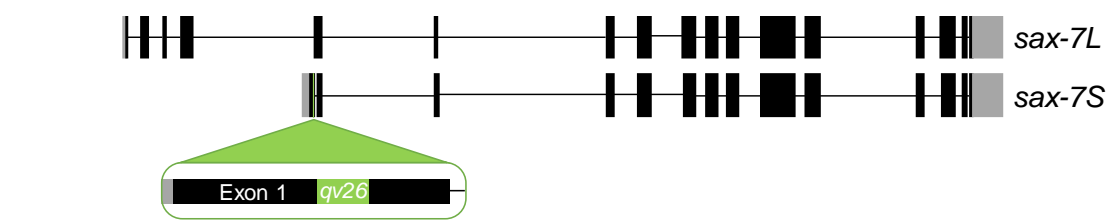

qv26 insertion sequence

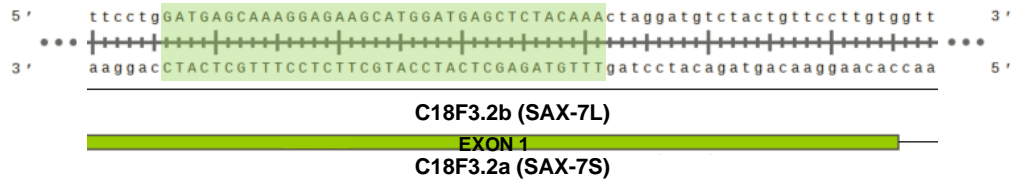

Figure $\mathbf{S 1}$ 


\section{D qv31 sfGFP::SAX-7S}

5. caaccttcatattcctgctaggatgtctactgttccttgtgtcggatcgctactacacaatgagcaaaggagagaactttcactggagttgtcccaattc

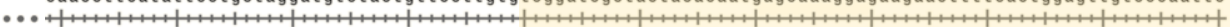
3. gttggagtataagacgatcctacagatgacaagaacacagcctagcgatgatgtgttactcgttectcttctgaaagtgacctcaacaggttaag

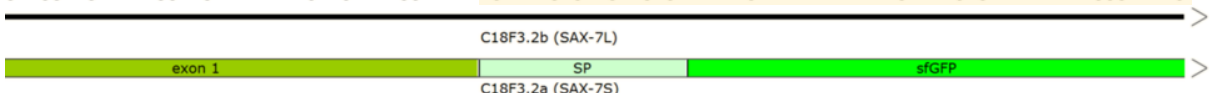
C18F3.2a (SAX-7S)

ttgttgaat agatggtgatgttaatgggcacaatttctgtccgtggagagggtgaaggtgatgctacaaacggaaactcaccct aaatt tatt tgcactactgga

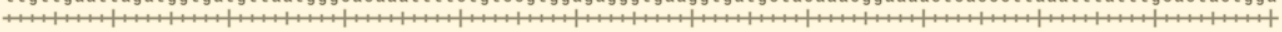
aacaact taatctaccactacaattaccogtgtt taaagacaggcacctctcccact tccactacgatgtttgcctttgagtgggaatttaaataaacgtgatgacct C18F3.2b (SAX-7L) C18F3.2a (SAX-7S)

aactacctgttccgtggccaacact tgtcactactctgacctatggtgttcaatgct ttccegttatccggatcacatgaacggcatgact tt tcaagagtgccat

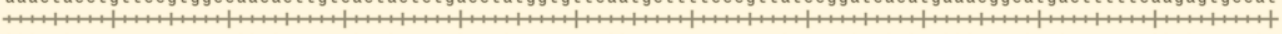
tttgatggacaaggcaccggttgtgaacagtgatgagactggataccacaagttacgaaagggcaataggcctagtgtactttgccgtactgaaaagttctcacggta

\begin{tabular}{cc}
\hline C18F3.2b (SAX-7L) \\
\hline SfGFP & C18F3.2a (SAX-7S)
\end{tabular}

gccogaggttatgtacaggaacgcactatatcttcaaagatgacgggacctacaagacgcgtgctgaagtcaagtt gaaggtgataccttgttaatcgtatcgagt

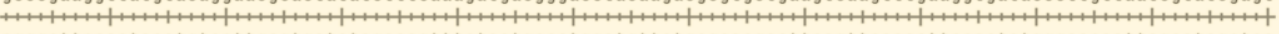
cgggcttccaatacatgtcettgegtgatatagaagttctactgccetggatgttctgcgcacgacttcagttcaacttccactatgggaacaattagcatagctca

C18F3.2b (SAX-7L) S18F3.2a (SAX-7S)

taagggtat tgatt t aaagaagatggaacattcttggacacaaactcgagt acaactttaactcacacaatgtatacatcacggcagacaaacaaagaatggaatc

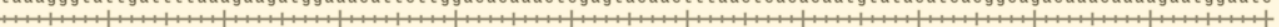
attcccataactaaattectctaccttgtaagacctgtgttgagctcatgttgaattgagtgtgttacatatgtagtgccgtctgttgtttcttaccttag C18F3.2b (SAX-7L) C18F3.2a (SAX-7S)

aa agct a act tcaaa t tcgccacaacgt tgaagatggt tccgt tcaactagcagaccat tat caacaaat actccaat tggcgat ggccctgt cct t t accagacaa

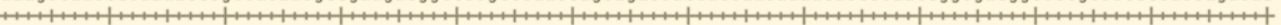
tt cgattgaagtttaagcggtgttgcaact tctaccaaggcaagttgatcgtctggtaatagttgtttatgaggttaaccgctaccgggacaggaaatggtctgtt C18F3.2b (SAX-7L) C18F3.2a (SAX-7S)

ccat tacctgtcgacacaatctgtcctt tcgaaagatcccaacgaaaagcgtgaccacatggtccttcttgagt tgtaactgctgctgggat tacacatggcatggat

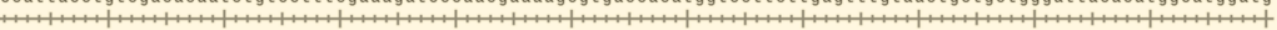
ggtaatggacagctgtgttagacaggaaagctttctagggttgctt ttcgcactggtgtaccaggaagaactcaaacattgacgacgaccctaatgtgtaccgtacct C18F3.2b (SAX-7L)

agctctacaagttagttcattcatttectttagggcacattgttcttcactatgttaatgaaaggattcaagtcggatcgctactacacatgtac 3

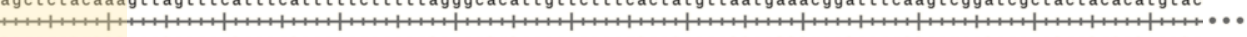
tcgagatgttcaatcaagtaagtaaaagaaa tcccgtgtaacaagaagtgatacaattactttgcctaagttcagcctagcgatgatgtgtacatg 5

SFGFP

Figure S1 
A

\begin{tabular}{c|c|c|c} 
& + & sax-7(qv30) & sax-7(nj48) \\
\hline $\mathbf{n}$ & 34 & 40 & 42 \\
\hline Egl worms & $9 \%$ & $8 \%$ & $5 \%$
\end{tabular}

B

\begin{tabular}{c|c|c|c} 
& + & sax-7(qv30) & sax-7(nj48) \\
\hline $\mathbf{n}$ & 386 & 440 & 389 \\
\hline Dead embryos & $0 \%$ & $1 \%$ & $0.5 \%$
\end{tabular}

C Brood size

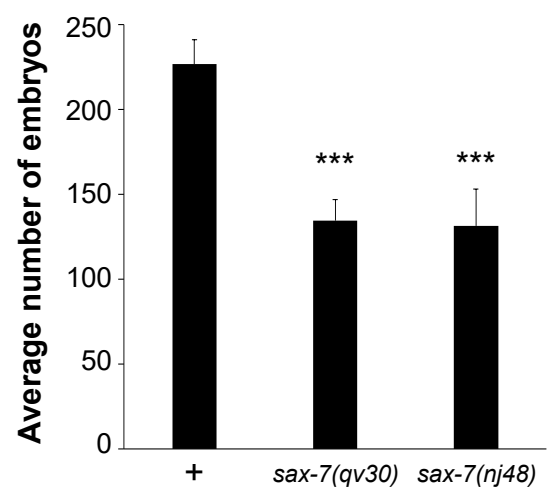

Figure S2 Desse-Bénard 
A

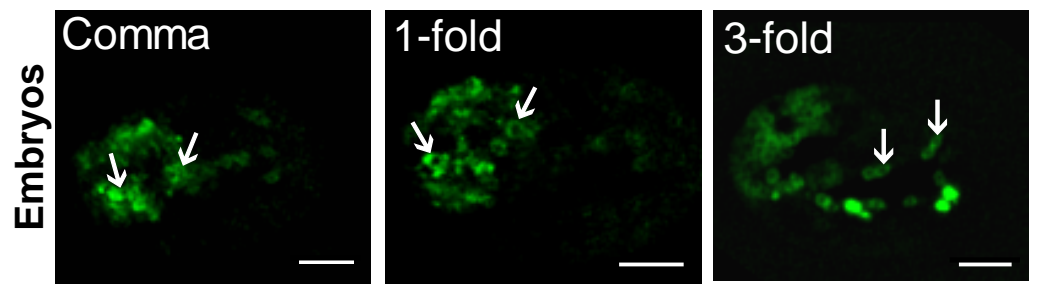

B

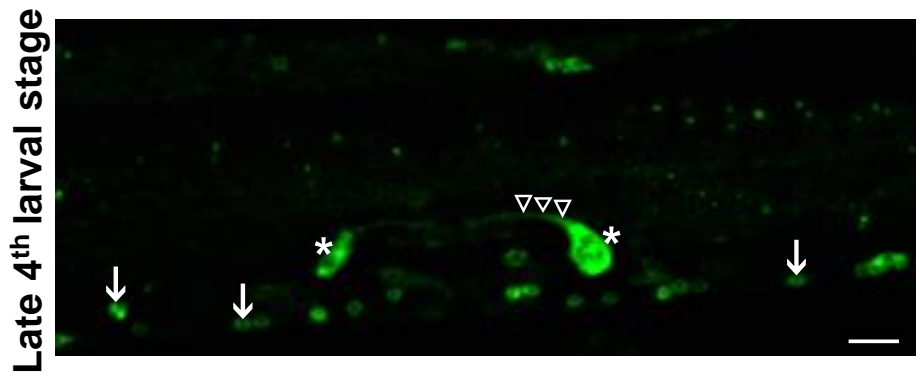

Figure S3

Desse-Bénard 